\title{
Analysis of Magnetic and Non Magnetic Nanoparticles With Newtonian/non-newtonian Base Fluids Over a Nonlinear Stretching Sheet
}

kanwal Jabeen ( $\square$ kanwaljabeen44@yahoo.com )

University of Engineering and Technology https://orcid.org/0000-0002-7546-1034

Muhamad Mushtaq

University of Engineering and Technology

Rana MUhammad Akram

University of Engineering and Technology

Original Article

Keywords: Magnetic \& non- Magnetic Nanoparticles, Casson nanofluid, water(Newtonian) and Sodium Alginate(non-Newtonian) base fluids, mixed convectional boundary layer flow

Posted Date: August 21st, 2020

DOI: https://doi.org/10.21203/rs.3.rs-58843/v1

License: (c) (i) This work is licensed under a Creative Commons Attribution 4.0 International License.

Read Full License 


\title{
Analysis of Magnetic and non Magnetic Nanoparticles with Newtonian/non-Newtonian Base Fluids Over a Nonlinear Stretching Sheet
}

\author{
K. Jabeen ${ }^{*}$ M. Mushtaq ${ }^{\dagger}$ and R. M. Akram Muntazir $\ddagger$ \\ Dpt of Math, UET, Lahore- Pakistan.
}

August 13, 2020

\begin{abstract}
Unsteady mixed convectional boundary layer flow of Casson nanofluid having magnetic $\left(\boldsymbol{F e}_{\mathbf{3}} \boldsymbol{O}_{\mathbf{4}}\right)$ and nonmagnetic $\left(\mathrm{Al}_{\mathbf{2}} \mathrm{O}_{\mathbf{3}}\right)$ nanoparticles suspension within two different types of base fluids, water(Newtonian) and Sodium Alginate(non-Newtonian), which incorporates viscous and ohmic dissipation effects over a permeable nonlinear stretching sheet with magnetic field effects. Some suitable non-dimensional similarity transformations are applied to convert the governing PDEs into a system of nonlinear coupled ODEs and then solved by differential transformation method(DTM) association with Pade-approximation. To validate the present results for limited cases, a comparison was made with previously existing literature and found to be in well agreement. The impact of radiation, internal heat sink/ source, viscous and ohmic dissipation was discussed for magnetic and non-magnetic nanofluid categories by tabular and graphical demonstrations. We have presented the tabular results of various emerging parameters to discuss the nature of skin friction, Nusselt and Sherwood numbers. It was also observed that performance of non-Newtonian(Sodium Alginate) fluid in heat and mass transfer is better than Newtonian(water) based fluid but no major difference was seen in heat and mass transfer when comparison was made with Magnetic and Non-magnetic nanoparticles.
\end{abstract}

Keywords: Magnetic \& non- Magnetic Nanoparticles, Casson nanofluid, water(Newtonian) and Sodium Alginate(non-Newtonian) base fluids, mixed convectional boundary layer flow

\section{HIGHLIGHTS}

- The Comparison of Newtonian \& non-Newtonian base fluid for magnetic \& non-magnetic nanoparticles was studied.

- Skin friction co-efficient, heat and mass fluxes were obtained.

- The impact of radiation, internal heat sink/ source, viscous and ohmic dissipation was observed for magnetic \& non-magnetic nanofluid categories.

- The variation of Schmidth number and chemical reaction reduces the thickness of concentration profiles.

- The velocity profile thickness depreciates for increasing magnetic field, porosity and unsteady parameter. These parameters help to control the flow rate.

\section{Introduction}

A fluid is a special kind of matter having no fixed shape that can deform easily because of external pressure. Mainly fluids are categorized as Newtonian and non-Newtonian fluids which have a lot of industrial examples such as food industry, polymer industry, paper production and many more extensive applications. On adverse condition there is no existing model that can fulfill all the needs of physical characteristics of non-Newtonian fluids. Several models are proposed to overcome this deficiency such as Maxwell, Williamson, Viscoelastic

\footnotetext{
*kanwaljabeen44@yahoo.com

${ }^{\dagger}$ mmushtaq@uet.edu.pk

‡akrammuntazir@gmail.com
} 
,Walter B- Model etc. For better and comprehensive interpretation of non-Newtonian fluids, Casson Model was presented in 1959 [1]. Casson model has shear thinning properties which is supposed to have infinite viscosity at zero shear rate, a yield stress, below which no flow occurs and a zero viscosity at an infinite shear rate. If the yield stress is greater than the shear stress then it would be referred as a solid but if the yield stress is smaller than the shear stress, the fluid will start to flow. Casson Model was fascinated by many researchers and scientists due to this unique property. Blood, Ketchup, concentrated juices, jellies etc are common examples of Casson fluid. This fluid has ability to capture complex rheological properties of a fluid as compared with other simplified fluid Models like power law 2nd \& 3rd third grade fluid models etc.

With an expanding universal race, industrial and engineering developments require efficient energy utilization and its implementation. For this purpose industries needs to evolve progressive heat transfer fluids with consequently higher thermal conductivities. Water, oil, glycol etc typically possess lower thermal conductivity which is a great barrier in the thermal reforms and applications. To overcome this deficiency, Maxwell initially initiated the idea of using small size solid particles in the regular base fluid to enlarge the thermal conductivity. Although it was observed that suspension of large and micro or milli- sized particles creates disruption technically. For example (i): extensive drop in pressure (ii): pipelines erosion (iii): clogging micro channels of devices (iv): faster settling time (v): absorption of surfaces. Keeping a constant view on the hindrances Choi and Eastman [2 - 4] not only introduced the concept of enhancing thermal conductivity using nano/microsized particles but also explicitly verified that the inclusion of nano-sized particles in the regular fluid increases the thermal conductivity. This mixture composed of nano-particles with traditional fluid which was named as Nanofluids. Nanofluids was preferred due to many scientific reasons like nanofluids are more stable as they have longer suspension time, reduce the demands of pumping power, having the capacity of saving energy etc. Although nano-particles have numerous applications such as improving the efficiency of diesel generators, automotive, air conditioning, power plant cooling, solar water heating, drilling thermal storage etc.

Generally nanoparticles are formed by $\mathrm{TiO}_{2}, \mathrm{AIN}, \mathrm{Al}_{2} \mathrm{O}_{3}, \mathrm{Cu}, \mathrm{SiO}$, and graphite. On comparison, we can find these nanoparticles possess elevated thermal conductivity than conventional base fluid. Eastman et al. [5] further observed that $\mathbf{4 0 \%}$ thermal conductivity can be enhanced by adding $\mathbf{C u}(\mathbf{1 0 n m})$ particles into an ethylene glycol. With the passage of time many researchers reported that by adding $(\mathbf{1}-\mathbf{5}) \%$ nanoparticles, more than $\mathbf{2 0 \%}$ thermal conductivity can be increased in ordinary fluids. Boungiorno's model [6] is the most popular model who studied thermal conductivity of nanofluids experimentally. Further the same author investigated the Brownian diffusion and thermophorsis effects for convective transports in nanofluids and proposed an analytical model [7] and concluded that Brownian motion and thermophorsis would be used to expand thermal conductivity in base fluid. Much related work can be seen in $[\mathbf{8}-\mathbf{1 2}]$. When magnetic nanoparticle, in the presence of magnetic field are added to base fluid, form a nanofluid subclass. Since, they frequently respond to applied magnetic field. By adding various types of magnetic nanoparticles, ferrofluids can be synthesised. Ferrofluid has many practical applications within a scientific field [13]. Some common examples are sensors, magnetic based devices, biomedical engineering and medicines. Casson fluid flow towards MHD nonlinear stretching sheet with slip effects was studied by [14]. An analytical steady state solution of nanofluid flow of sisko model was presented by [15]. There are basically three subclasses that state how material is influenced with magnetic field:

- Diamagnetism: water, copper, quartz, lead, etc are diamagnetic. They are very less influenced by the Magnetic flux.

- Paramagnetism: sodium, platinum, oxygen, iron oxide $(\boldsymbol{F e} \boldsymbol{O})$, are paramagnetic. They are much more affected by field of force than diamagnetism

- Ferromagnetism: iron gadolinium, magnetite $\left(\mathrm{Fe}_{3} \mathrm{O}_{4}\right), \mathrm{CoFe}_{3} \mathrm{O}_{4}$, nickel, and $\mathrm{MnBi}$ are strenuously affected by the magnetic field and highly polarized in the magnetic field direction and interestingly they retain their state of polarization even when field is disconnected.

All above type of fluids are together known as ferromagnetic fluids [21]. Ferrofluids [13] are normally referred as magnetic fluids. Ferrofluids have nicely proven their effectiveness in magnetically targeting the drug delivery to specific part of human body, targeting cancer cells or tumors, monitoring brains activities, removal of toxins for the treatment of cancer etc. Ferrofluid as magnetic nanoparticle has tremendous medical applications as they have ability to reach even in small capillaries of human body. Another useful application of ferrofluid is magnetic ink which is less expensive and high speed for silent printers. Ferrofluids can also be used to control the underground flow via externally applied magnetic field. In [16] field dependent swirling viscous ferrofluid along a porous rotating disk was studied. The results were obtained for electrically non-conducting fluids. Some more interesting work relating effectiveness of ferrofluids can be found in $[\mathbf{1 7}-\mathbf{2 2}]$.

A study of magnetic\& non-magnetic nanoparticles with Newtonian \& non-Newtonian based fluids was firstly discussed by Hakeem et. al in [24]. By numerical investigation they concluded that magnetic nanoparticles are more influenced by the magnetic field as compared by non-magnetic nanoparticles. They also claimed that non-Newtonian based nanofluids have greater skin friction and Nusselt number than Newtonian based nanofluids. Same author [25] investigates the $\mathbf{2}-\boldsymbol{D}$ steady convective flow and heat transfer with two sorts of 
Newtonian(Water) and non-Newtonian(Sodium Alginate) base fluids along a flat plate. Non magnetic $\left(\mathrm{Al}_{2} \mathrm{O}_{3}\right)$ and magnetite $\left(\mathrm{Fe}_{3} \mathrm{O}_{4}\right)$ nanoparticles were added in base fluids and discussed the consequences of thermal radiation and slip conditions. Despite of already existing research, heat transfer fluids are still needed to improve thermal enhancement. Due to thermophysical properties of $\mathrm{Al}_{2} \mathrm{O}_{3}$ (non-magnetic) and $\mathrm{Fe}_{3} \mathrm{O}_{4}$ (magnetite) are considered to be most suitable option and hence selected for present research.

\section{$3 \quad$ Formulation of Flow Model}

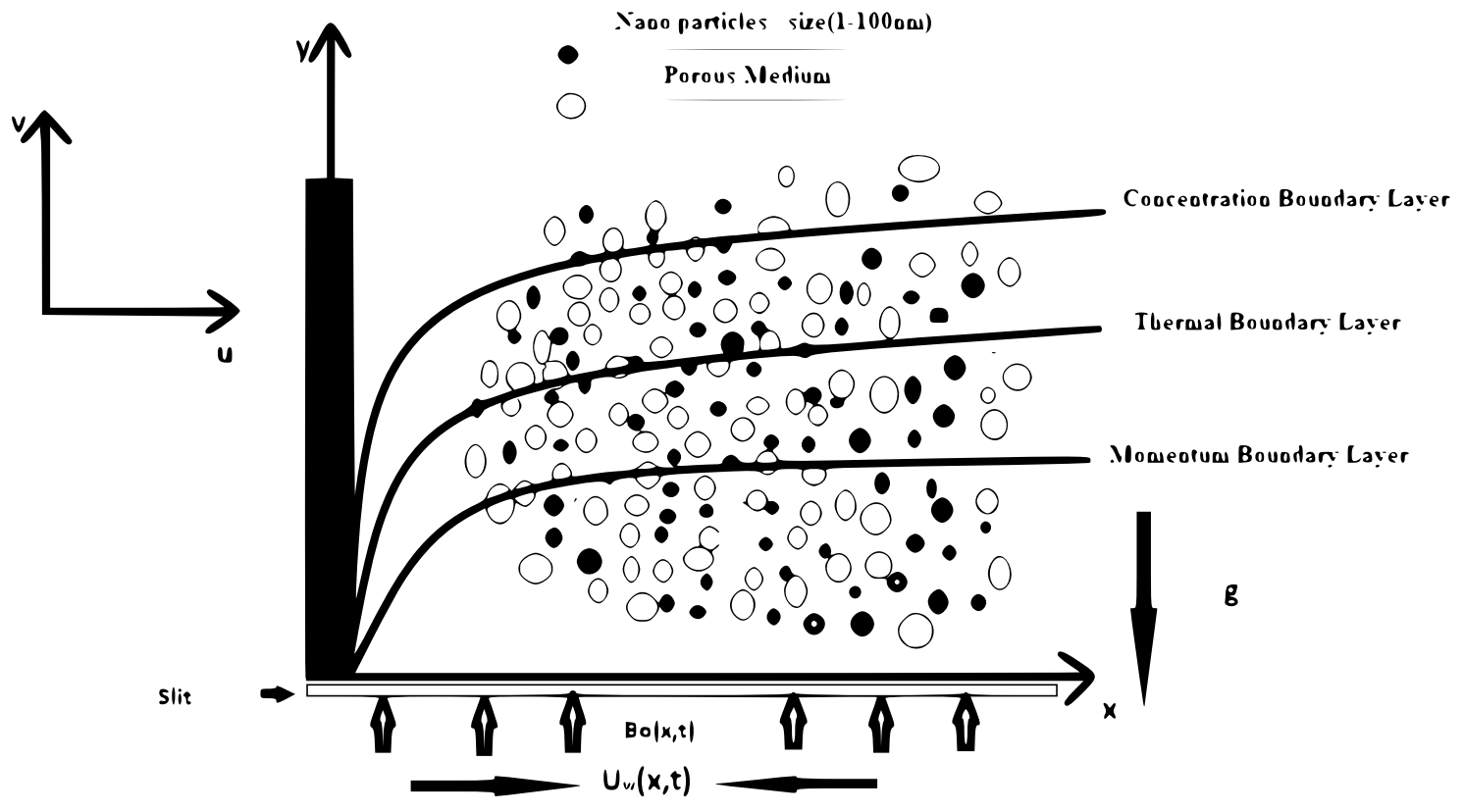

Figure 1: Physical Interpretation of Flow Model

The non-dimensional form of conservation equations have attained worth attention due to various transport mechanisms in the flow of nanofluids. Although non-dimensionalization can have many advantages, some of which are listed as below: $(i)$ Analysis of any system without looking at their material properties. (ii) Flow parameters are easy to understand. (iii) Shape and size of the geometry can be more generalized in nondimensionalization. (iv) Physical problem can be guessed by experiment.

Mixed convectional, unsteady, laminar and incompressible flow of water and sodium alginate based Casson fluid having magnetic and non-magnetic nanoparticles is considered along a nonlinear stretched sheet fixed in a penetrable medium. The base fluids as well as magnetic \& non-magnetic nanofluids are presumed in thermal equilibrium. The $\mathrm{x}$-axis is in the direction of stretched sheet and y-axis is normal to the sheet (see fig.1). The velocity of the sheet is

$\boldsymbol{U}_{\boldsymbol{w}}=\frac{c_{1} \boldsymbol{x}^{n}}{1-\gamma t}$ where $\boldsymbol{c}_{1}, \gamma \geq \mathbf{0}$ are constants, $\mathrm{t}$ is the time and $\boldsymbol{n}>\mathbf{0}$ is representing the nonlinear parameter of the sheet.

A magnetic flux of intensity $\boldsymbol{B}_{\mathbf{0}}$ is enforced perpendicular to a sheet and defined as

$B(x, t)=B_{0} x^{\frac{n-1}{2}}(1-\gamma t)^{\frac{-1}{2}}$

Conversion laws under the boundary layer estimations in a dimensional mode are [28]:

$$
\frac{\partial u}{\partial x}+\frac{\partial v}{\partial y}=0
$$

$$
\begin{aligned}
\frac{\partial u}{\partial t}+u \frac{\partial u}{\partial x}+v \frac{\partial u}{\partial y}=\frac{\mu_{n f}}{\rho_{n f}}\left(1+\frac{1}{\beta}\right) \frac{\partial^{2} u}{\partial y^{2}} & -\frac{\sigma_{n f}}{\rho_{n f}} B^{2}(x, t)-\frac{\mu_{n f}}{\rho_{n f}}\left(1+\frac{1}{\beta}\right) \frac{u \phi_{1}}{K} \\
& +g\left[\frac{\left(\sigma B_{T}\right)_{n f}}{\rho_{n f}}\left(T-T_{\infty}\right)+\frac{\left(\sigma B_{c}\right)_{n f}}{\rho_{n f}}\left(C-C_{\infty}\right)\right]
\end{aligned}
$$




$$
\begin{gathered}
\frac{\partial T}{\partial t}+u \frac{\partial T}{\partial x}+v \frac{\partial T}{\partial y}=\alpha_{n f} \frac{\partial^{2} T}{\partial y^{2}}+{\frac{\mu_{n f}}{\left(\rho C_{p}\right)}}_{n f}\left(1+\frac{1}{\beta}\right)\left(\frac{\partial u}{\partial y}\right)^{2}-\frac{1}{\left(\rho C_{p}\right)_{n f}} \frac{\partial q_{r}}{\partial y} \\
+\frac{1}{\left(\rho C_{p}\right)}{ }_{n f} q^{\prime \prime \prime}, \\
\frac{\partial C}{\partial t}+u \frac{\partial C}{\partial x}+v \frac{\partial C}{\partial y}=D_{m} \frac{\partial^{2} C}{\partial y^{2}}+D_{1} \frac{\partial^{2} T}{\partial y^{2}}-k_{o}\left(C-C_{\infty}\right),
\end{gathered}
$$

where velocity components are $\mathrm{u}$ and $\mathrm{v}$, effective thermal conductivity is $\boldsymbol{k}_{\boldsymbol{n} \boldsymbol{f}}$, dynamic viscosity is $\boldsymbol{\mu}_{\boldsymbol{n} \boldsymbol{f}}$, effective density is $\boldsymbol{\rho}_{\boldsymbol{n} \boldsymbol{f}}$, effective heat capacity is $\left(\rho \boldsymbol{C}_{\boldsymbol{p}}\right)_{\boldsymbol{n} \boldsymbol{f}}$, and $\boldsymbol{\alpha}_{\boldsymbol{n} \boldsymbol{f}}=\frac{\boldsymbol{k}_{\boldsymbol{n}}}{\left(\rho \boldsymbol{C}_{\boldsymbol{p}}\right)_{\boldsymbol{n}}}$ is the thermal diffusivity of the nano-fluids as mentioned below $[\mathbf{2 4 , 2 5}]$

$$
\begin{aligned}
& \mu_{n f}=\frac{\mu_{f}}{(1-\phi)^{2.5}}, \quad \rho_{n f}=(1-\phi) \rho_{f}+\phi \rho_{s} \\
& \frac{k_{n f}}{k_{f}}=\frac{k_{s}+2 k_{f}-2 \phi\left(k_{f}-k_{s}\right)}{k_{s}+2 k_{f}+\phi\left(k_{f}-k_{s}\right)}, \quad\left(\rho C_{p}\right)_{n f}=(1-\phi)\left(\rho C_{p}\right)_{f}+\phi\left(\rho C_{p}\right)_{s},
\end{aligned}
$$

where $\phi$ is volume fraction of the nano-particles. Strength of magnetic field induction is B, $\boldsymbol{\beta}$ is Casson parameter , $\boldsymbol{B}_{\boldsymbol{T}}, \boldsymbol{B}_{\boldsymbol{c}}$ are thermal and concentration expansion coefficients, $\boldsymbol{q}_{\boldsymbol{r}}$ is radiation heat flux , mass diffusion co-efficients $\boldsymbol{D}_{\boldsymbol{m}}$, and mean temperature and $\boldsymbol{D}_{\mathbf{1}}$ is thermal diffusion co-efficients and $\boldsymbol{k}_{\mathbf{0}}$ is the chemical reaction rate. Also $B(x, t)=\frac{B_{0} x^{\frac{n-1}{2}}}{(1-\gamma t)^{\frac{1}{2}}}$ and $\boldsymbol{K}(x, t)=\frac{k_{p}(1-\gamma t)}{x^{n-1}}$ are variable magnetic field and variable permeability parameters respectively.

The above governing equations $(\mathbf{1} \mathbf{- 4})$ are associated with the Bc's as follows:

$$
\left\{\begin{array}{l}
\mathrm{y}=0: \\
t<0: u=v=0, T=T_{\infty}, C=C_{\infty}, \\
t \geqslant 0: u(x, t)=U_{w}(x, t)+N_{1} \nu_{f}\left(1+\frac{1}{\beta}\right) \frac{\partial u}{\partial y}, \\
k \frac{\partial T}{\partial y}=-h_{w}\left(T_{w}-T\right), D_{B} \frac{\partial C}{\partial y}=-h_{s}\left(C_{w}-C\right) \\
\mathrm{y} \rightarrow \infty: \\
u=0, T \rightarrow T_{\infty}, C \rightarrow C_{\infty}
\end{array}\right.
$$

In boundary conditions $N_{1}=\frac{N_{0}(1-\gamma t)^{-\frac{1}{2}}}{x^{\frac{n-1}{2}}}, \quad h_{w}=h_{0} x^{\frac{n-1}{2}}(1-\gamma t)^{-\frac{1}{2}}, h_{s}=h_{1} x^{\frac{n-1}{2}}(1-\gamma t)^{-\frac{1}{2}}$ are convective heat and mass transfer with $N_{0}, h_{0}$ and $h_{1}$ are constants $T_{w}=T_{\infty}+\frac{c_{2} x^{2 n-1}}{(1-\gamma t)^{2 n-1}}, C_{w}=$ $C_{\infty}+\frac{c_{3} x^{2 n-1}}{(1-\gamma t)^{2 n-1}}$ where $c_{2}$ and $c_{3}$ are temperature and concentration references.

The radioactive heating flux in the temperature equation is calculated and simplified by the Rosseland approximation i.e

$q_{r}=-\frac{4 \sigma^{*}}{3 k^{*}} \frac{\partial T^{4}}{\partial y}$

The heat variation in the flow is supposed to be less enough so $\boldsymbol{T}^{4}$ is taken a linear relation of temperature ignoring terms having higher power index while expanding $\boldsymbol{T}^{\mathbf{4}}$ at $\boldsymbol{T}_{\boldsymbol{\infty}}$ is obtained as, $[\mathbf{2 8}, \mathbf{3 2}, \mathbf{3 3}]$

$$
T^{4}=T_{\infty}^{4}+4 T_{\infty}^{3}\left(T-T_{\infty}\right)+6 T_{\infty}^{2}\left(T-T_{\infty}\right)^{2}+\ldots
$$

we get,

Also $\boldsymbol{q}^{\prime \prime \prime}$ is modeled as $[\mathbf{3 2}, \mathbf{3 3}]$

$$
T^{4} \cong 4 T T_{\infty}^{3}-3 T_{\infty}^{3}
$$

$q^{\prime \prime \prime}=\frac{U_{w}}{x} \frac{k_{n f}}{\nu n f}\left[A\left(T_{w}-T_{\infty}\right) f^{\prime}+B\left(T-T_{\infty}\right)\right]$

Introducing similarity transformation, $[28]$

$\eta=\sqrt{\frac{c_{1}(n+1)}{2 \nu_{f}(1-\gamma t)}} y, \quad \psi(x, y, t)=x^{\frac{n+1}{2}} f(\eta) \sqrt{\frac{2 \nu_{f} c_{1}}{(n+1)(1-\gamma t)}}, \quad \theta(\eta)=\frac{T-T_{\infty}}{T_{w}-T_{\infty}}, \quad h(\eta)=\frac{C-C_{\infty}}{C_{w}-C_{\infty}}$

The system of transformed non-linear ODEs are:

$$
\left(1+\frac{1}{\beta}\right) f^{\prime \prime \prime}+A_{1}\left[f f^{\prime \prime}-\frac{2 n}{n+1} f^{\prime 2}-S\left(\frac{2}{n+1} f^{\prime}+\frac{\eta}{n+1} f^{\prime \prime}\right)\right]-M \frac{A_{1}}{A_{2}} f^{\prime}-K_{p}\left(1+\frac{1}{\beta}\right) f^{\prime}
$$




$$
\begin{array}{r}
+\tau\left(\frac{2}{n+1}\right) \frac{A_{1} A_{3}}{A_{2}}\left(\theta+\frac{A_{4}}{A_{3}} N h\right)=0 \\
\left(1+\frac{4}{3} R\right) \theta^{\prime \prime}+\operatorname{Pr} \frac{A_{6}}{A_{5}}\left[f \theta^{\prime}-\frac{2(2 n-1)}{n+1} f^{\prime} \theta-S\left(\frac{2(2 n-1)}{n+1} \theta+\frac{\eta}{n+1} \theta^{\prime}\right)\right]+ \\
\operatorname{EcPr} \frac{A_{6}}{A_{5} A_{7}}\left(1+\frac{1}{\beta}\right) f^{\prime \prime 2}+A_{1}\left(\frac{2}{n+1} A^{*} f^{\prime}+\frac{2}{n+1} B^{*} \theta\right)=0 \\
\left.h^{\prime \prime}+S c\left[f h^{\prime}-\frac{2(2 n-1)}{n+1} f^{\prime} h-S\left(\frac{2(2 n-1)}{n+1} h+\frac{\eta}{n+1} h^{\prime}\right)\right)+S_{r} \theta^{\prime \prime}-K_{r} h\right]=0
\end{array}
$$

Also the transformed BC's are:

$$
\begin{aligned}
& \left\{\begin{array}{l}
f(0)=0 ; f^{\prime}(0)=1+\delta\left(1+\frac{1}{\beta}\right) f^{\prime \prime}(0) ; f^{\prime}(\infty)=0 \\
\theta^{\prime}(0)=-B i_{1}(1-\theta(0)) ; \theta(\infty)=0 ; h^{\prime}(0)=-B i_{2}(1-h(0)) ; h(\infty)=0 \\
f^{\prime \prime}(0)=\alpha_{1} ;(\text { say }) \theta(0)=\alpha_{2} ;(\text { say }) h^{\prime}(0)=\alpha_{3},(\text { say })
\end{array}\right. \\
& \left(\alpha_{i} s \quad i=1 \text { to } 3 \text { are constant to determine }\right)
\end{aligned}
$$

where dimensionless parameters in $(\mathbf{7}-\mathbf{9})$ equations are

$M=\frac{2 \sigma B_{0}^{2}(x)}{(n+1) c_{1} \rho}{ }_{f}, \quad K_{p}=\frac{(2 \nu)_{f}}{(n+1) c_{1} k p}, \quad S=\frac{\gamma}{c_{1}} x^{n-1}$, Magnetic, porosity and unsteady parametes

$\tau=\frac{\lambda}{R e^{2}}, \quad \lambda=\frac{g\left(B_{T}\right)_{f}\left(T_{w}-T_{\infty}\right) x^{3}}{\nu_{f}^{2}}$ thermal buoyancy parameter, $\frac{1}{R^{2}}=\frac{\nu_{f}^{2}}{U_{w}^{2} x^{2}}$ local reynold number, $\boldsymbol{N}=\frac{\left(\boldsymbol{B}_{c}\right)_{s}\left(\boldsymbol{C}_{\boldsymbol{w}}-\boldsymbol{C}_{\infty}\right)}{\left(\boldsymbol{B}_{T}\right)_{f}\left(\boldsymbol{T}_{\boldsymbol{w}}-\boldsymbol{T}_{\infty}\right)}$, , Buoyancy ratio parameter

$\boldsymbol{P r}=\frac{\nu_{f}\left(\rho C_{p}\right)_{f}}{(k)_{f}}, \quad \boldsymbol{E} \boldsymbol{c}=\frac{\rho_{f} U_{w}^{2}}{\left(\boldsymbol{T}_{w}-\boldsymbol{T}_{\infty}\right)\left(\rho C_{p}\right)_{f}}, \quad \boldsymbol{R}=\frac{4 \sigma^{*} T_{\infty}^{3}}{\boldsymbol{k}^{*}(\boldsymbol{k})_{n f}}$, Prandtl, Eckret and Radiation parameter ,$\delta=N_{0} \sqrt{\frac{(n+1) c_{1} \nu_{f}}{2}}$ is the slip parameter,

$S c=\frac{(\nu)_{f}}{D_{m}}, \quad S r=\frac{D_{1}\left(T_{w}-T_{\infty}\right)}{(\nu)_{f}\left(C_{w}-C_{\infty}\right)}, \quad K_{r}=\frac{2 k_{r}(1-\gamma t)}{c_{1}(n+1) x^{n-1}}$

Schmidth and Soret numbers and chemical reaction parameter respectively.

$\boldsymbol{B} i_{1}=-\frac{h_{0}}{k} \sqrt{\frac{2 \nu_{f}}{c_{1}(n+1)}}$ and $\boldsymbol{B} i_{1}=\frac{h_{1}}{D_{B}} \sqrt{\frac{2 \nu_{f}}{c_{1}(n+1)}}$ are the Biot numbers.

Also, $\boldsymbol{A}_{\mathbf{1}}$ to $\boldsymbol{A}_{\mathbf{7}}$ defined as below are the nanofluid parameters.

$$
\begin{aligned}
& A_{1}=(1-\phi)^{2.5}\left[(1-\phi)+\phi \frac{\rho_{s}}{\rho_{f}}\right] \\
& A_{2}=(1-\phi)+\phi \frac{\rho_{s}}{\rho_{f}}, \\
& A_{3}=(1-\phi)+\phi \frac{\left(\rho B_{T}\right)_{s}}{\left(\rho B_{T}\right)_{f}} \\
& A_{4}=(1-\phi)+\phi \frac{\left(\rho B_{c}\right)_{s}}{\left(\rho B_{c}\right)_{f}} \\
& A_{5}=\frac{k_{n f}}{k_{f}}=\frac{k_{s}+2 k_{f}-2 \phi\left(k_{f}-k_{s}\right)}{k_{s}+2 k_{f}+\phi\left(k_{f}-k_{s}\right)} \\
& A_{6}=(1-\phi)+\phi \frac{\left(\rho C_{p}\right)_{s}}{\left(\rho C_{p}\right)_{f}}, \\
& A_{7}=(1-\phi)^{2.5}\left[(1-\phi)+\phi \frac{\left(\rho C_{p}\right)_{s}}{\left(\rho C_{p}\right)_{f}}\right]
\end{aligned}
$$


The significant physical parameters of engineering concerns are dimensionless skin friction coefficient $\boldsymbol{C}_{\boldsymbol{f} \boldsymbol{x}}$ , Nusselt numbers $\boldsymbol{N}_{\boldsymbol{u} \boldsymbol{x}}$ and sherwood number $\boldsymbol{S} \boldsymbol{h}_{\boldsymbol{x}}$ that physically indicates shear stress, rate of heat \& mass transfer respectively and are defined as under:

$C_{f x}=\frac{\tau_{w x}}{\rho_{f} U_{w}^{2}}, \quad N_{u x}=\frac{x q_{w}}{k_{f}\left(T_{w}-T_{\infty}\right)}$ and $\quad S h_{x}=\frac{x q_{s}}{D_{B}\left(C_{w}-C_{\infty}\right)}$

where $\boldsymbol{\tau}_{\boldsymbol{w} \boldsymbol{x}}$ is the shear stress at the sheet along x-axis :

$\tau_{w x}=\mu_{n f}\left(\frac{\partial u}{\partial y}\right)_{y=0}$,

$\boldsymbol{q}_{\boldsymbol{w}}$ and $\boldsymbol{q}_{\boldsymbol{s}}$ are surface heat \& mass fluxes defined as:

$q_{w}=-k_{n f}\left(\frac{\partial T}{\partial y}\right)_{y=0}+q_{r}, \quad q_{s}=-D_{B}\left(\frac{\partial C}{\partial y}\right)_{y=0}$

Upon fixing the local Reynold number $\boldsymbol{R} \boldsymbol{e}_{\boldsymbol{x}}=\frac{\boldsymbol{x} U_{\boldsymbol{w}}}{\boldsymbol{\nu}_{\boldsymbol{f}}}$ and using the above defined relations we have

$$
\begin{aligned}
& \left(R e_{x}\right)^{\frac{1}{2}} C_{f x}=\left(1+\frac{1}{\beta}\right) \sqrt{\frac{n+1}{2}} \frac{f^{\prime \prime}(0)}{(1-\phi)^{2.5}} \\
& \left(R e_{x}\right)^{\frac{-1}{2}} N_{u x}=-\frac{k_{n f}}{k_{f}} \sqrt{\frac{n+1}{2}}\left(1+\frac{4}{3} R\right) \theta^{\prime}(0) \\
& \left(R e_{x}\right)^{\frac{-1}{2}} S h_{x}=-\sqrt{\frac{n+1}{2}} h^{\prime}(0)
\end{aligned}
$$


Table-1: Thermophysical Properties of Nanofluids

\begin{tabular}{|c|c|c|c|c|}
\hline & $\rho \quad\left(\frac{k g}{m^{3}}\right)$ & $C_{p} \quad\left(\frac{J}{k g K}\right)$ & $k\left(\frac{W}{m K}\right)$ & $\sigma\left(\frac{1}{\Omega m}\right)$ \\
\hline Water(newtonian) & 997.1 & 4179 & 0.613 & $5.5 \times 10^{-6}$ \\
\hline SodiumAlginate (non - newtonian $)$ & 989 & 4175 & 0.6376 & $2.6 \times 10^{-4}$ \\
\hline $\mathrm{Al}_{2} \mathrm{O}_{3}($ non - magnetic $)$ & 3970 & 765 & 40 & $35 \times 10^{6}$ \\
\hline $\mathrm{Fe}_{3} \mathrm{O}_{4}($ magnetic $)$ & 5180 & 670 & 9.7 & $2.5 \times 10^{4}$ \\
\hline
\end{tabular}

Table-2:- Computation of $C_{f x}$ when $\phi=0.0, K p=S=E_{1}=\delta=N=\tau=0, n=1$

\begin{tabular}{|cccccc|}
\hline \multicolumn{5}{c}{$\begin{array}{c}\sqrt{\frac{n+1}{2}}\left(1+\frac{1}{\beta}\right) f^{\prime \prime}(0) \\
\text { Pade }[8,8]\end{array}$} \\
\hline$\infty$ & 0 & -1.0042 & 1.00000 & 1.000 & 1.000156518 \\
5 & & -1.0954 & -1.09544 & -1.0955 & -1.095409494 \\
1 & & -1.4142 & -1.41421 & -1.4144 & -1.414231210 \\
$\infty$ & 10 & -3.3165 & -3.31662 & -3.3166 & -3.316593053 \\
5 & & -3.6331 & -3.63318 & -3.6332 & -3.633194220 \\
1 & & -4.6904 & -4.69042 & -4.6904 & -4.690368068 \\
$\infty$ & 100 & -10.049 & -10.04987 & -10.0499 & -10.04985548 \\
5 & & -11.0091 & -11.00909 & -11.0091 & -11.00924228 \\
1 & & -14.2127 & -14.21267 & -14.2127 & -14.21252529 \\
\hline
\end{tabular}

Table-3:- Comparison of $C_{f x}$ when $\phi=0, K p=S=E_{1}=\delta=N=\tau=0, n=1$

\begin{tabular}{|c|cccc|}
\hline & \multicolumn{3}{|c}{$\sqrt{\frac{n+1}{2}}\left(1+\frac{1}{\beta}\right) f^{\prime \prime}(0)$} & Pade $[8,8]$ \\
$S$ & 9 & 14 & {$[27,28]$} & Present Outcomes \\
\hline 0.8 & -1.261512 & -1.261042 & -1.2610 & -1.261711535 \\
1.2 & -1.378052 & -1.377722 & -1.377850 & -1.377980614 \\
\hline
\end{tabular}


Table-4:- Calculation of $N_{u x}$ when $\phi=0, \beta \rightarrow 0, B i_{1} \rightarrow 0 \quad K p=S=E_{1}=\delta=N=\tau=E c=R=0, n=1$

\begin{tabular}{|c|cccc|}
\hline & \multicolumn{5}{|c|}{$\sqrt{\frac{n+1}{2}} \theta^{\prime}(0)$} & Pade $[8,8]$ \\
$P r$ & {$[3]$} & {$[12]$} & {$[27,28]$} & Present Results \\
\hline 0.72 & 0.8086 & 0.8086 & 0.8086 & 0.8087872052 \\
1 & 1.0000 & 1.00000 & 1.0000 & 1.0000 \\
3 & 1.9237 & 1.9237 & 1.9237 & 1.923737976 \\
10 & 3.7207 & 3.7207 & 3.7206 & 3.7201550382 \\
100 & 12.2940 & 12.3004 & 12.2939 & 12.28675446 \\
\hline
\end{tabular}

Table-5:- Calculation of $-f^{\prime \prime}(0)$ when $M=S c=2, \mathrm{~N}=0, \mathrm{n}=1, \delta=R=K_{r}=0.5, K_{p}=E_{1}=R=K_{r}=0.5$,

$$
\operatorname{Pr}=0.72 \phi=E c=S r=A^{*}=B^{*}=S=\tau=0.2
$$

\begin{tabular}{|c|c|c|c|c|c|c|c|c|c|}
\hline \multirow[b]{2}{*}{$M$} & \multirow[b]{2}{*}{$S$} & \multirow[b]{2}{*}{$K_{p}$} & \multirow[b]{2}{*}{$\phi$} & \multirow[b]{2}{*}{$\boldsymbol{\beta}$} & \multirow[b]{2}{*}{$\delta$} & \multicolumn{2}{|l|}{ Water } & \multicolumn{2}{|l|}{ SodiamAlginate } \\
\hline & & & & & & $\mathrm{Al}_{2} \mathrm{O}_{3}$ & $\mathrm{Fe}_{3} \mathrm{O}_{4}$ & $\mathrm{Al}_{2} \mathrm{O}_{3}$ & $\mathrm{Fe}_{3} \mathrm{O}_{4}$ \\
\hline 1 & & & & & & -0.802902083 & -0.813376195 & -0.78047890 & -0.78680841 \\
\hline 2 & & & & & & -0.940385416 & -0.948970023 & -0.801047943 & -0.829442709 \\
\hline 3 & & & & & & -1.051107790 & -1.054149593 & -1.0162769409 & -1.021951737 \\
\hline & 0 & & & & & -0.898237212 & -0.909219437 & -0.799010325 & -0.843839491 \\
\hline & 0.5 & & & & & -0.906923041 & -0.9300919284 & -0.821132745 & -0.876241098 \\
\hline & 1 & & & & & -0.936919443 & -0.961916095 & -0.8610342781 & -0.931826394 \\
\hline & & 0.5 & & & & -0.955087231 & -0.989667263 & -0.918934952 & -0.963298145 \\
\hline & & 1.0 & & & & -0.963891376 & -0.9968732411 & -0.926461023 & -0.976201984 \\
\hline & & 1.5 & & & & -0.975028471 & -1.0032765167 & -0.939826547 & -0.982813621 \\
\hline & & & 0.05 & & & -0.94985276 & -0.9550823418 & -0.932891746 & -0.942781338 \\
\hline & & & 0.10 & & & -1.152635142 & -1.1762892330 & -0.956173982 & -0.987398124 \\
\hline & & & 0.20 & & & -1.433094378 & -1.475028732 & -1.001982376 & -1.018329374 \\
\hline & & & & 1 & & - & - & -0.635167698 & -0.653598714 \\
\hline & & & & 3 & & - & - & -0.858794213 & -0.877540271 \\
\hline & & & & 5 & & - & - & -0.9234188715 & -0.935428753 \\
\hline & & & & & 0.5 & -0.949856438 & -0.955087563 & -0.804646782 & -0.827986092 \\
\hline & & & & & 1.0 & -0.633958601 & -0.648609267 & -0.603298516 & -0.638601184 \\
\hline & & & & & 1.5 & -0.485122965 & -0.498139430 & -0.469614678 & -0.478362390 \\
\hline
\end{tabular}


Table-6:- Calculation of $-\theta^{\prime}(0)$ when $M=S c=2, \mathrm{~N}=0, \mathrm{n}=1, \delta=R=K_{r}=0.5, \quad K_{p}=E_{1}=R=K_{r}=0.5$,

\begin{tabular}{|c|c|c|c|c|c|c|c|c|c|c|}
\hline \multirow[b]{2}{*}{$M$} & \multirow[b]{2}{*}{$S$} & \multirow[b]{2}{*}{$\phi$} & \multirow[b]{2}{*}{$B i_{1}$} & \multirow[b]{2}{*}{$E c$} & \multirow[b]{2}{*}{$R$} & \multirow[b]{2}{*}{$A^{*}$} & \multicolumn{2}{|l|}{ Water } & \multicolumn{2}{|c|}{ Sodiam Alginate } \\
\hline & & & & & & & $\mathrm{Al}_{2} \mathrm{O}_{3}$ & $\mathrm{Fe}_{3} \mathrm{O}_{4}$ & $\mathrm{Al}_{2} \mathrm{O}_{3}$ & $\mathrm{Fe}_{3} \mathrm{O}_{4}$ \\
\hline 1 & & & & & & & 1.154557289 & 1.150407159 & 0.992488675 & 0.97928572 \\
\hline 2 & & & & & & & 1.646441981 & 1.621627742 & 1.246690498 & 1.234497730 \\
\hline 3 & & & & & & & 2.373566773 & 2.132720498 & 1.827523683 & 1.683298123 \\
\hline & 0 & & & & & & 0.474489126 & 0.490382912 & 0.462091283 & 0.4673940139 \\
\hline & 0.5 & & & & & & 0.359069783 & 0.368298346 & 0.331983592 & 0.348391274 \\
\hline & 1 & & & & & & 0.226604872 & 0.247987265 & 0.212273853 & 0.237198344 \\
\hline & & 0.05 & & & & & 0.8087872052 & 0.807293586 & 0.793928732 & 0.782494853 \\
\hline & & 0.10 & & & & & 0.839485120 & 0.819327543 & 0.803891254 & 0.798194302 \\
\hline & & 0.20 & & & & & 0.9337821382 & 0.914274628 & 0.847918353 & 0.8293753057 \\
\hline & & & 0.5 & & & & 0.242252649 & 0.231937562 & 0.240127834 & 0.229827563 \\
\hline & & & 3.0 & & & & 0.981763521 & 0.972741273 & 0.958129472 & 0.942718394 \\
\hline & & & 10 & & & & 1.299823871 & 1.292375639 & 1.192846790 & 1.191873652 \\
\hline & & & & 0.2 & & & 0.893910472 & 0.882653891 & 0.8209459721 & 0.820218387 \\
\hline & & & & 0.4 & & & 0.921093723 & 0.902813654 & 0.852901873 & 0.830912845 \\
\hline & & & & 0.8 & & & 0.954508922 & 0.937082013 & 0.882930278 & 0.861087343 \\
\hline & & & & & 0.5 & & 0.692046521 & 0.67328656467 & 0.684087239 & 0.663921458 \\
\hline & & & & & 1.0 & & 0.755682834 & 0.739085208 & 0.710238659 & 0.709385670 \\
\hline & & & & & 1.5 & & 0.955082389 & 0.942840193 & 0.892356349 & 0.876304854 \\
\hline & & & & & & 0.2 & 0.786409371 & 0.780289274 & 0.764902841 & 0.729067469 \\
\hline & & & & & & 0.4 & 0.8849502446 & 0.860923741 & 0.849103845 & 0.812049856 \\
\hline & & & & & & 0.8 & 0.955082389 & 0.942840193 & 0.892356349 & 0.876304854 \\
\hline
\end{tabular}


Table-7:- Calculation of $-h^{\prime}(0)$ when $M=S c=2, \mathrm{~N}=0, \mathrm{n}=1, \delta=R=K_{r}=0.5, K_{p}=E_{1}=R=K_{r}=0.5$,

\begin{tabular}{|c|c|c|c|c|c|c|c|c|c|}
\hline \multicolumn{10}{|c|}{$\operatorname{Pr}=0.72 \phi=E c=S r=A^{*}=B^{*}=S=\tau=0.2$} \\
\hline \multirow[b]{2}{*}{$M$} & \multirow[b]{2}{*}{$S$} & \multirow[b]{2}{*}{$K_{r}$} & \multirow[b]{2}{*}{$S c$} & \multirow[b]{2}{*}{$S r$} & \multirow[b]{2}{*}{$B i_{2}$} & \multicolumn{2}{|l|}{ Water } & \multicolumn{2}{|c|}{ SodiamAlginate } \\
\hline & & & & & & $\mathrm{Al}_{2} \mathrm{O}_{3}$ & $\mathrm{Fe}_{3} \mathrm{O}_{4}$ & $\mathrm{Al}_{2} \mathrm{O}_{3}$ & $\mathrm{Fe}_{3} \mathrm{O}_{4}$ \\
\hline 1 & & & & & & 1.266144730 & 1.262348375 & 1.264578321 & 1.247829187 \\
\hline 2 & & & & & & 1.36189596 & 1.360983732 & 1.342983763 & 1.339017303 \\
\hline 3 & & & & & & 1.501674057 & 1.494156231 & 1.494814761 & 1.490126334 \\
\hline & 0 & & & & & 1.770897632 & 1.769988272 & 1.698736320 & 1.676737676 \\
\hline & 0.5 & & & & & 1.5389289711 & 1.5299082837 & 1.492768283 & 1.479092233 \\
\hline & 1 & & & & & 1.231971887 & 1.230989134 & 1.201927365 & 1.1987465321 \\
\hline & & 0.5 & & & & 2.043198218 & 2.033103291 & 1.987401132 & 1.9738821827 \\
\hline & & 1.0 & & & & 1.749035375 & 1.745606200 & 1.696366399 & 1.689233547 \\
\hline & & 1.5 & & & & 1.338345623 & 1.330836921 & 1.329421364 & 1.313361667 \\
\hline & & & 0.5 & & & 2.069774579 & 1.947610063 & 2.066288451 & 1.943674602 \\
\hline & & & 1.0 & & & 1.086243391 & 1.025408845 & 1.084415239 & 1.023514794 \\
\hline & & & 2 & & & 0.592069204 & 0.567448867 & 0.591282948 & 0.565671566 \\
\hline & & & & 0.2 & & 1.183989123 & 1.1567139839 & 1.170933563 & 1.1702983542 \\
\hline & & & & 0.4 & & 1.293268456 & 1.264469095 & 1.293641735 & 1.27341738 \\
\hline & & & & 0.8 & & 1.338345623 & 1.3224276812 & 1.319421364 & 1.302984567 \\
\hline & & & & & 0.5 & 0.449088267 & 0.4477882910 & 0.441834938 & 0.4409837422 \\
\hline & & & & & 3.0 & 1.158881903 & 1.159711984 & 1.129837367 & 1.1240924763 \\
\hline & & & & & 10 & 1.593398478 & 1.587920742 & 1.397628293 & 1.3798320128 \\
\hline
\end{tabular}



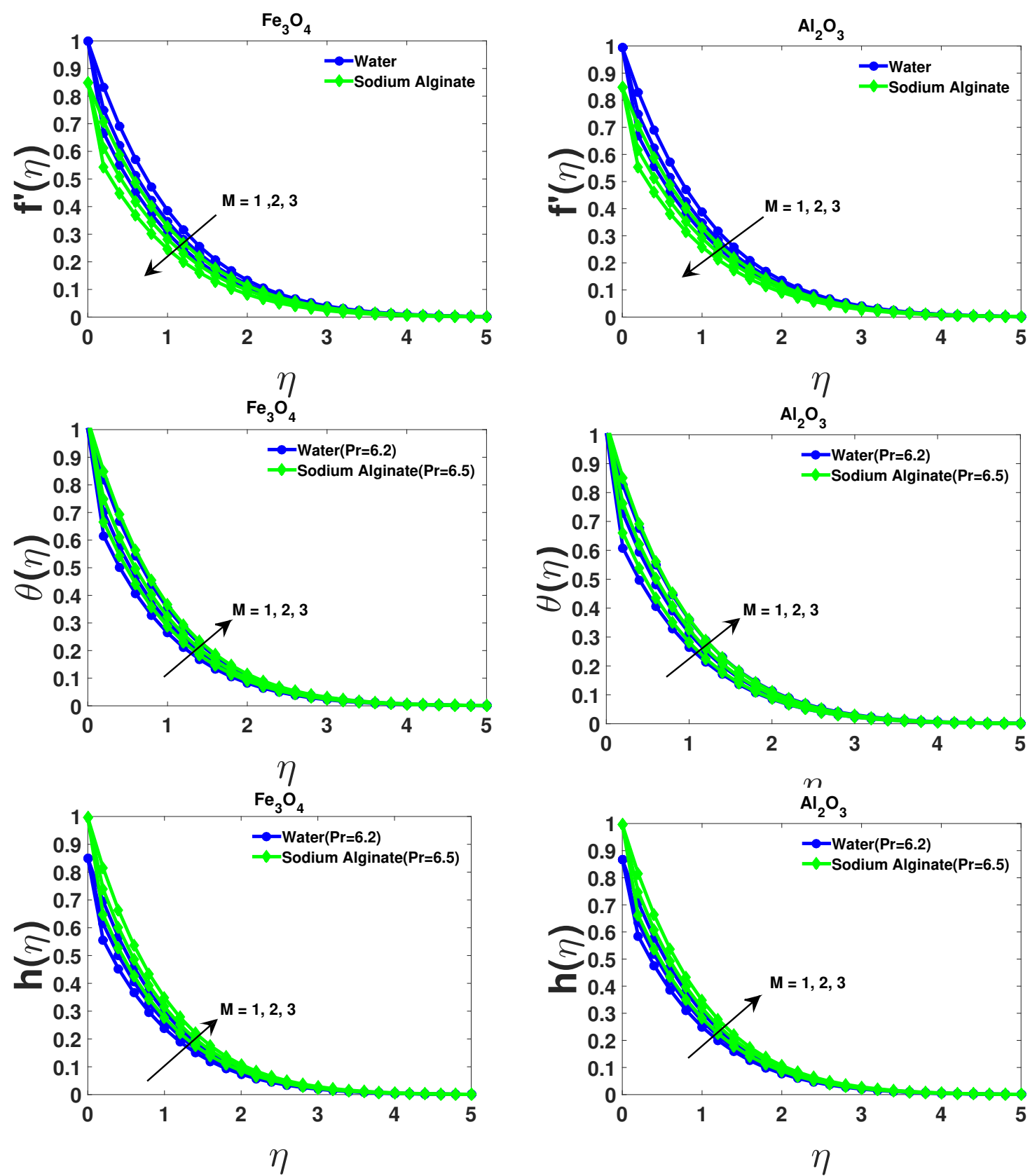

Figure 2: Effects of Magnetic field $\mathrm{M}$ for $\mathrm{Fe}_{3} \mathrm{O}_{4}$ and $\mathrm{Al}_{2} \mathrm{O}_{3}$ 

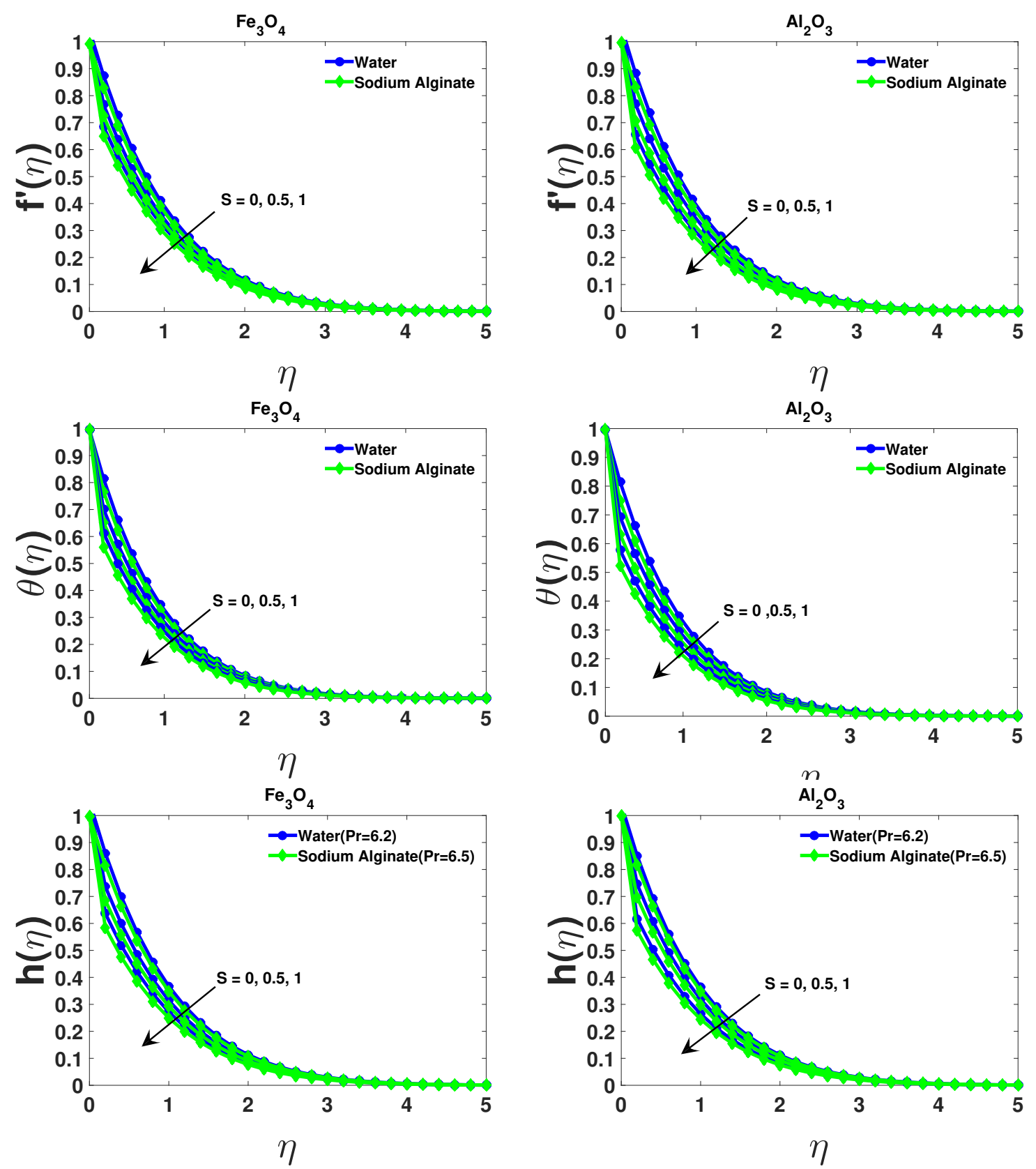

Figure 3: Effects of Unsteady parameter $\mathrm{S}$ for $\mathrm{Fe}_{3} \mathrm{O}_{4}$ and $\mathrm{Al}_{2} \mathrm{O}_{3}$
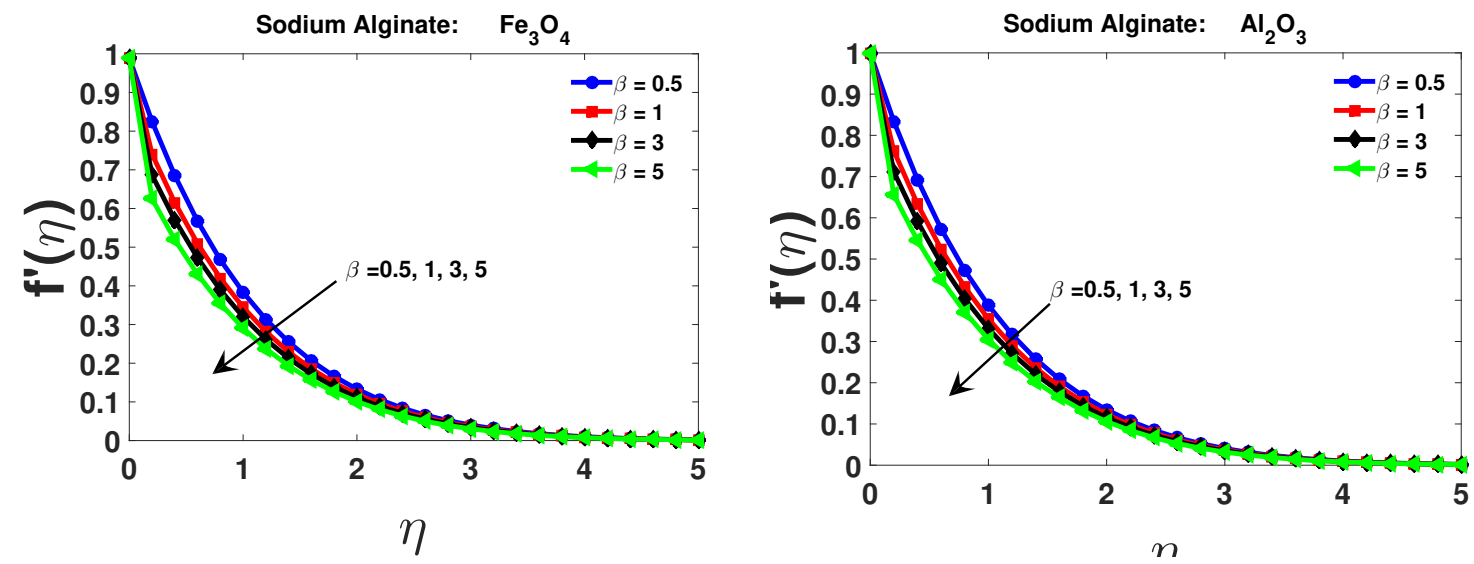

Figure 4: Effects of Casson Parameter $\beta$ for $\mathrm{Fe}_{3} \mathrm{O}_{4}$ and $\mathrm{Al}_{2} \mathrm{O}_{3}$ 

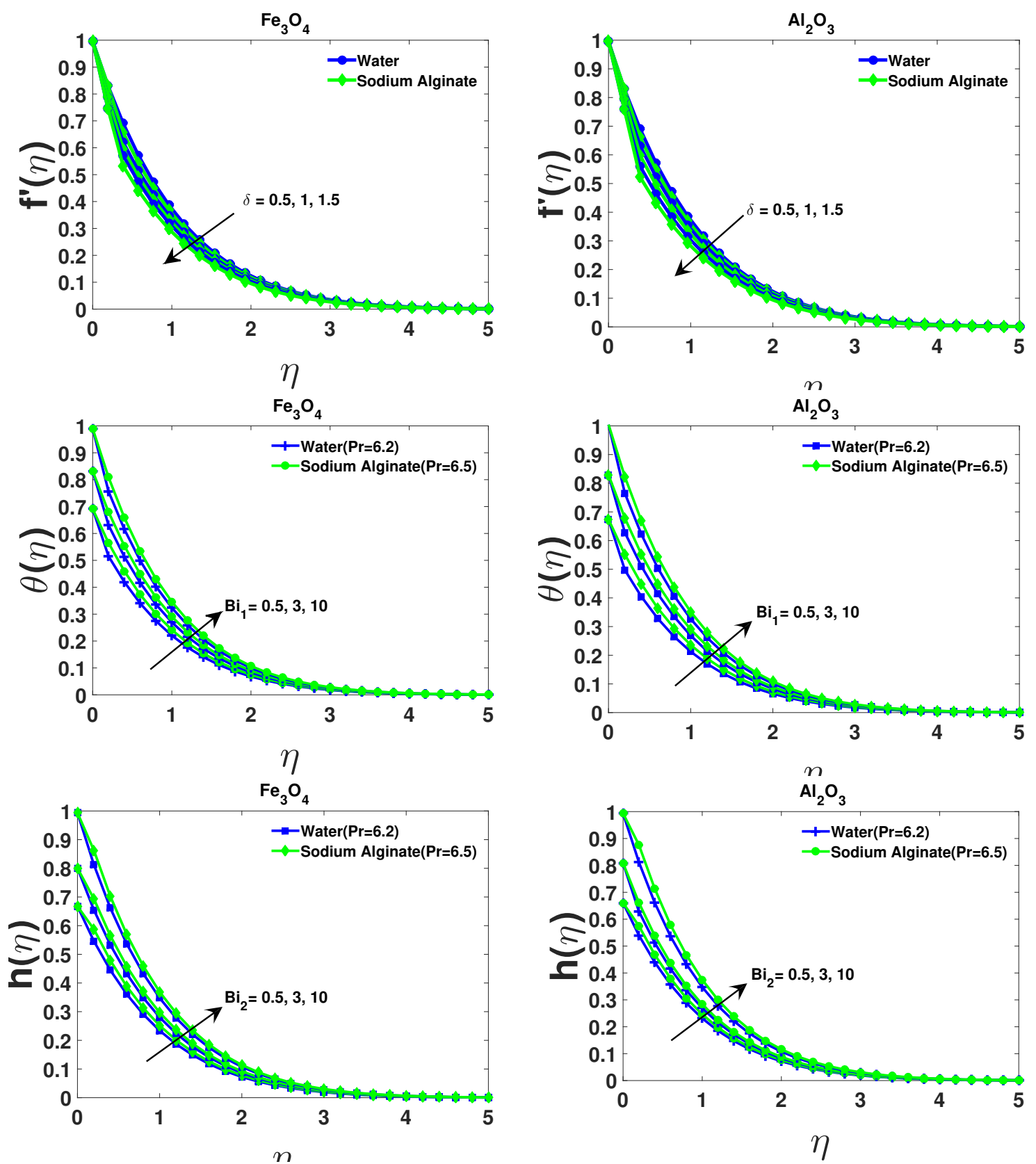

Figure 5: Effects of Slip Parameters $\delta, \mathrm{Bi}_{1}$ and $\mathrm{Bi}_{2}$ for $\mathrm{Fe}_{3} \mathrm{O}_{4}$ and $\mathrm{Al}_{2} \mathrm{O}_{3}$
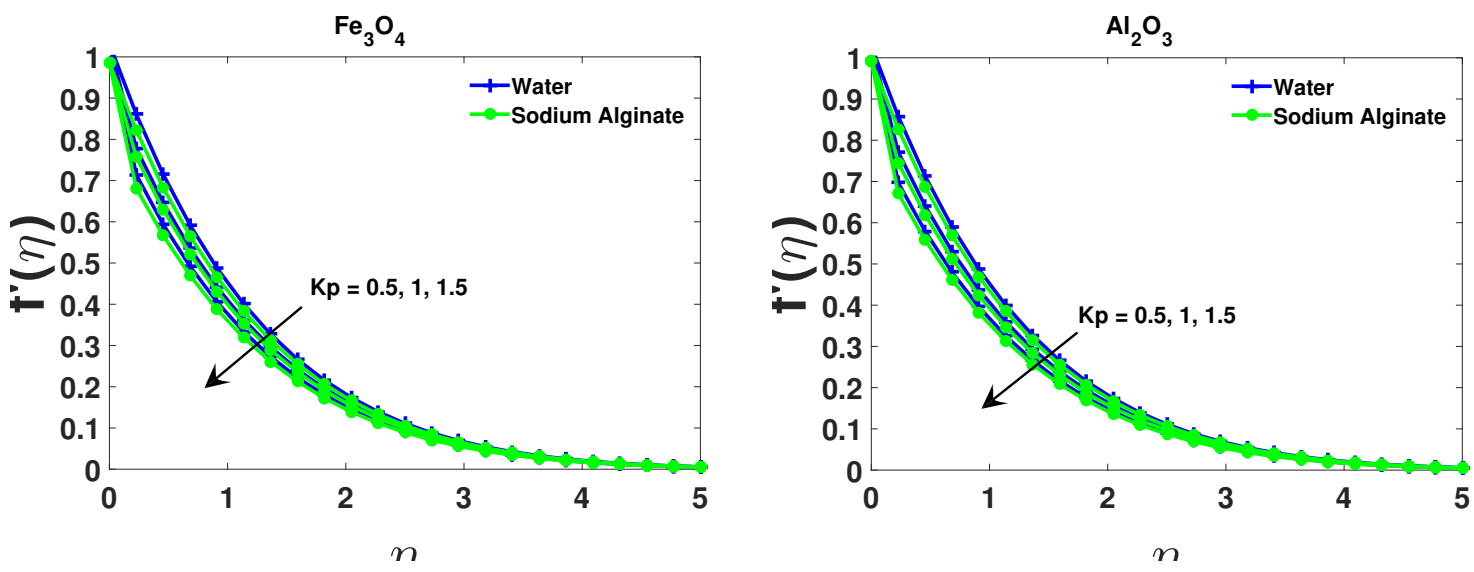

Figure 6: Effects of Porosity Parameters $\mathrm{Kp}$ for $\mathrm{Fe}_{3} \mathrm{O}_{4}$ and $\mathrm{Al}_{2} \mathrm{O}_{3}$ 

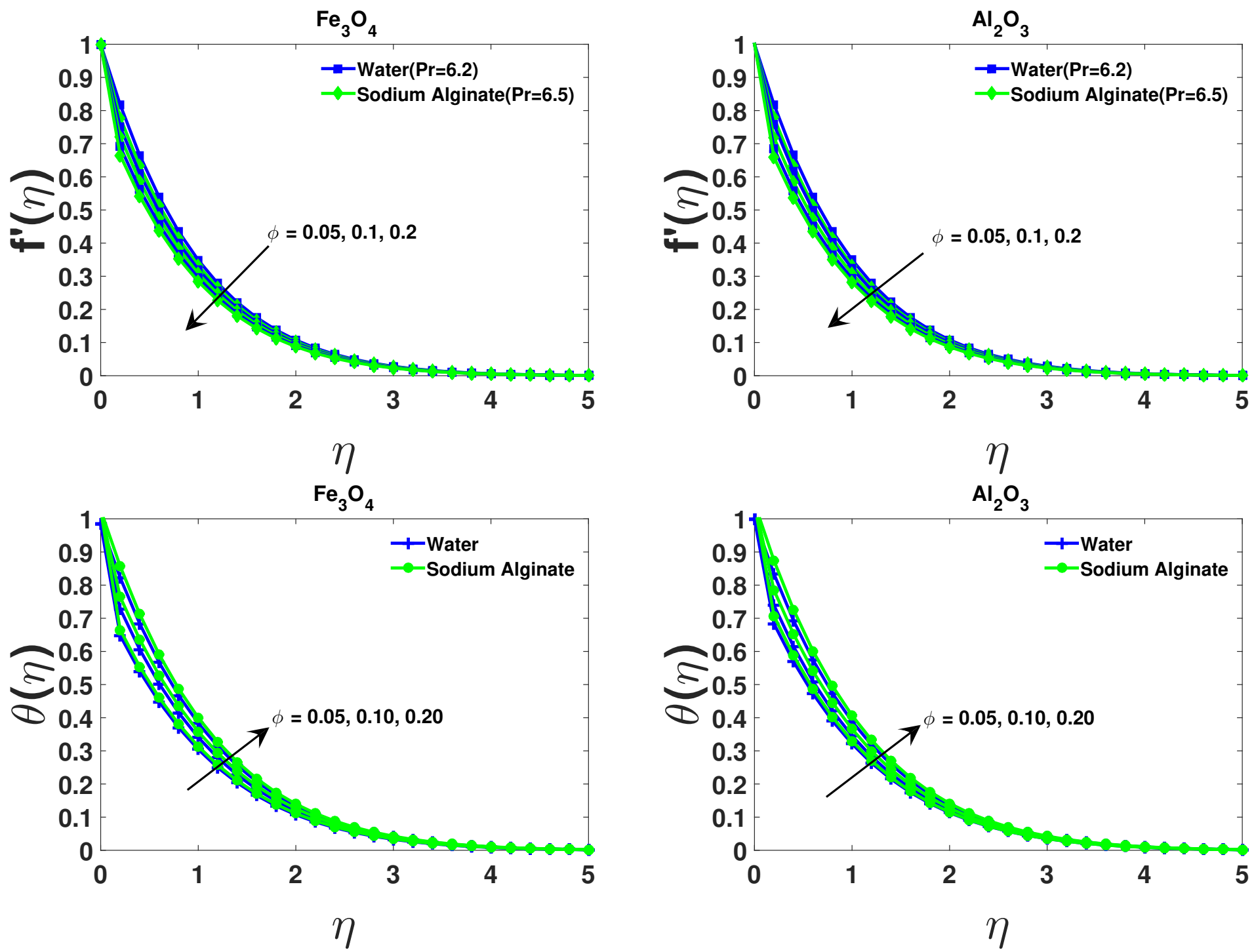

Figure 7: Effects of Volume fraction $\phi$ for $\mathrm{Fe}_{3} \mathrm{O}_{4}$ and $\mathrm{Al}_{2} \mathrm{O}_{3}$ 

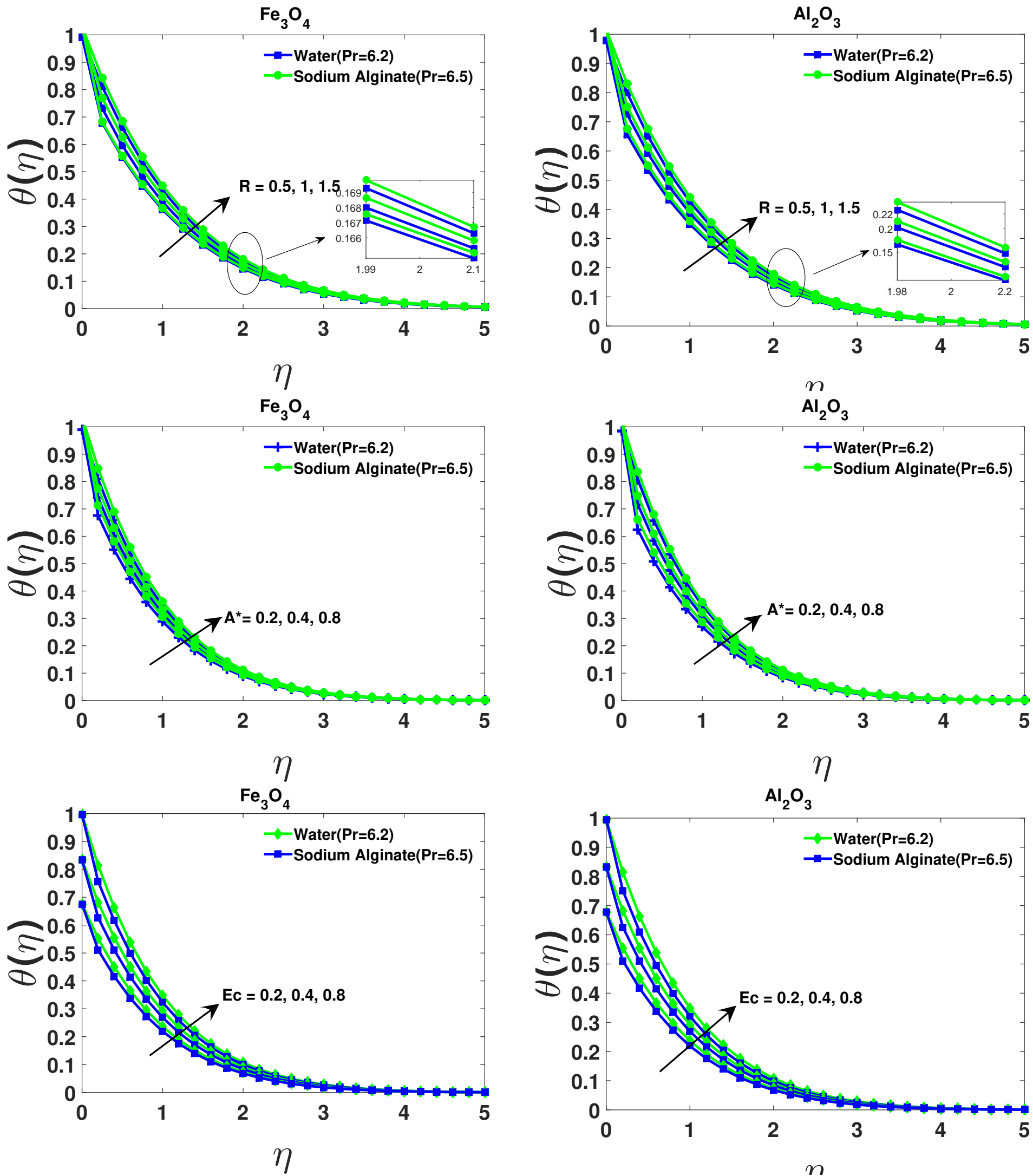

Figure 8: Effects of Radiation Parameters(R), heat sink / $\operatorname{source}\left(A^{*}\right)$ and Viscous dissipation(Ec) factors for $\mathrm{Fe}_{3} \mathrm{O}_{4}$ and $\mathrm{Al}_{2} \mathrm{O}_{3}$ 

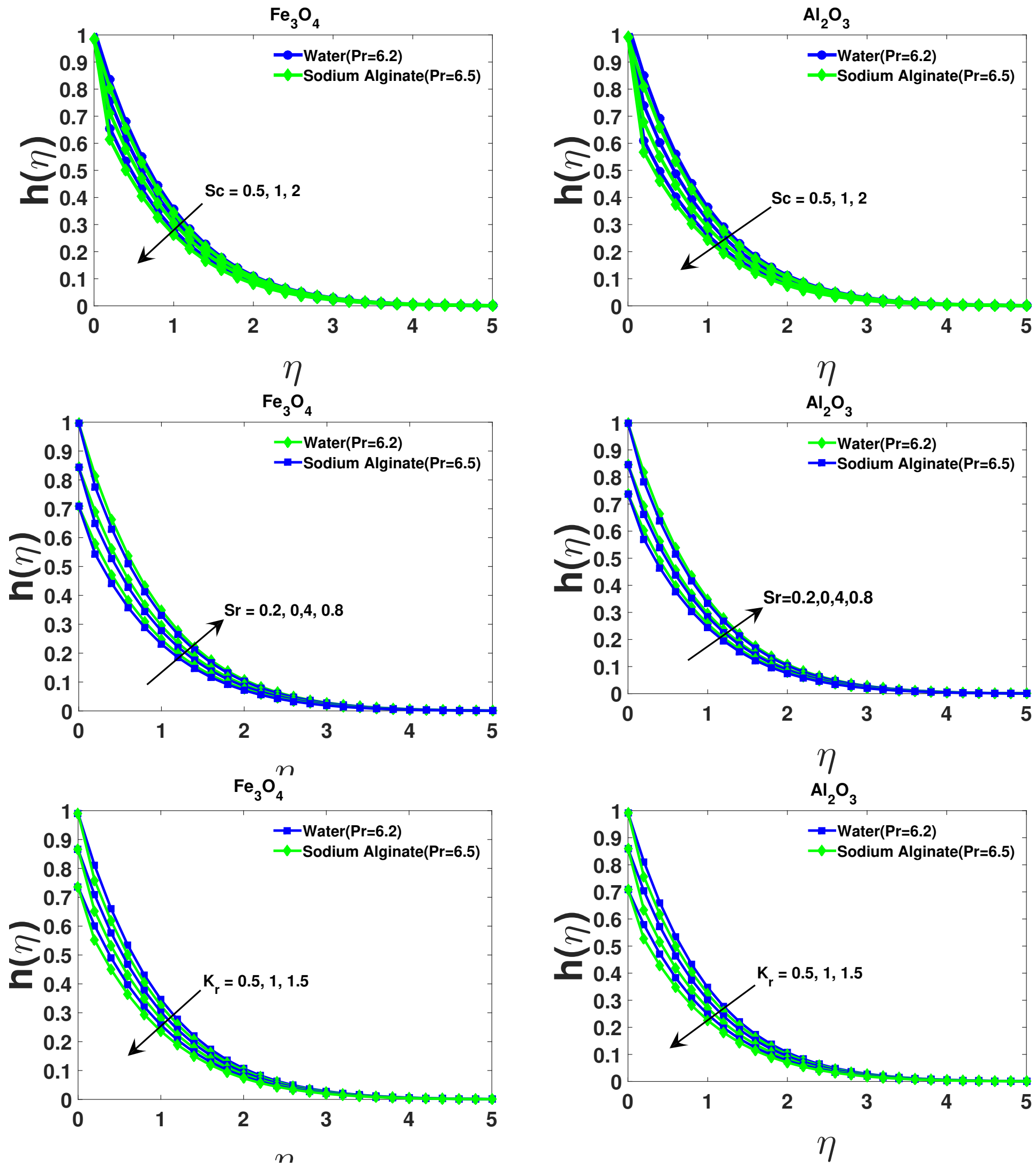

Figure 9: Effects of Sc, Sr and $\mathrm{K}_{r}$ for $\mathrm{Fe}_{3} \mathrm{O}_{4}$ and $\mathrm{Al}_{2} \mathrm{O}_{3}$ 


\section{Results and Discussion}

This work specifically scrutinized the nature of Newtonian(water) and non-Newtonian(sodium alginate) suspension containing magnetic $\mathrm{Fe}_{3} \mathrm{O}_{4}$ (making as ferrofluid) and non magnetic $\mathrm{Al}_{2} \mathrm{O}_{3}$ nanoparticles along-with mass \& heat transfer analysis. Time dependent, mixed convectional boundary layer flow of a casson nanofluid through non-linear stretching sheet in a porous media with effects of field of magnetic force, chemical reaction, viscous and ohmic dissipation was explored. For plausible understanding of physical problem, numerical computations are obtained to deliberate the impacts of various $\mathbf{2}-\boldsymbol{D}$ governing parameters on boundary layer profiles and results are presented in terms of tables and graphs. To validate the method the current outcomes are correlated with already published literature and displayed in the table $(\mathbf{2}-\mathbf{4})$. Table $\mathbf{2}$ and $\mathbf{3}$ shows the analogy of skin friction for variate values of Casson, magnetic field and unsteady parameter. These tables are in good agreement with $(\mathbf{9}, \mathbf{1 4}, \mathbf{1 7}, \mathbf{2 2}, \mathbf{2 7}, \mathbf{2 8})$. Table 4 highlights the comparison of $\boldsymbol{N}_{\boldsymbol{u x}}$ for different Prandtl number that revealed good agreement with $(\mathbf{3}, \mathbf{1 2}, \mathbf{2 7}, \mathbf{2 8})$. Table $[\mathbf{5}-\mathbf{7}]$ shows the variation of $C_{f x}, N_{u x}$ and $\boldsymbol{S} \boldsymbol{h}_{x}$ for various parameters for Newtonian/non-Newtonian base fluids alongside the magnetic \& non-magnetic particles.

$\mathrm{Fig}(2)$ indicates behavior of magnetic flux $(\mathrm{M})$ on the three profiles for magnetic $\left(\mathrm{Fe}_{3} \mathrm{O}_{4}\right.$-Magnetite) and non-magnetic $\left(\mathrm{Al}_{2} \mathrm{O}_{3}\right.$-Aluminium oxide $)$ nanoparticles, suspended on two distinct base fluids water $\left(\mathrm{H}_{2} \mathrm{O}\right)$ and other is sodium Alginate $(\boldsymbol{N a A l g})$ having Newtonian and non-Newtonian features respectively. In this figure we noticed the velocity profile for fluids having magnetic and non-magnetic nanoparticles decelerated but reverse trend was followed by the thermal and concentration profiles. This was due to the applied transverse magnetic field. As electrically transmitted nanoparticles generates Lorentz force which slower down the motion of nanoparticles in the boundary layer but it starts acceleration in the other two profiles. Fig(3) demonstrate the behavior of unsteady parameter S. As S enhances, all the three profiles started to decrease. It means less mass exchanged from the fluid to the boundary layer. It is quite obvious from physical situation of unsteadiness, when $\mathrm{S}$ rises, more heat is released by the sheet and hence the temperature starts diminishing. On the other hand one can say that for increasing values of $\mathrm{S}$ cooling rate is more agile. So, we can conclude that with the gradual increment of unsteady parameter $\mathrm{S}$, the thermal profile decreases due to less heat transferring from sheet to fluid. Almost similar behavior was seen for both magnetic and non-magnetic nano-fluids in newtonian and non-newtonian based liquids. Fig(4) precisely delineate the behavior observed by the Casson parameter $\boldsymbol{\beta}$. From figure it is obvious that by increasing the values of $\boldsymbol{\beta}$ the velocity of fluid started to decrease. This happens due to the inverse relationship of $\boldsymbol{\beta}$ with yield stress. When the values of $\boldsymbol{\beta}$ increases the yield stress decreases and hence decline the momentum profile for both geometries(magnetic \& non-magnetic nano-particles). Also, fluid plasticity is reduced by increasing the values of $\boldsymbol{\beta}$. In $\mathbf{F i g}(\mathbf{5})$ we had seen that velocity profile depreciate for increasing values of $\boldsymbol{\delta}$ but reverse effects was observed for thermal and concentration profiles. From a physical situation we have seen that as slip $(\boldsymbol{\delta}=\mathbf{0})$ occurs, the velocity of fluid that is adjoining to the sheet becomes smaller than the fluid's velocity $\perp$ to the sheet. Simply, increasing values of $\boldsymbol{\delta}$ allows more the fluid to flow around the sheet, this causes declination in momentum profile. In temperature profile $\boldsymbol{B} \boldsymbol{i}_{\mathbf{1}}>\mathbf{0 . 1}$ was considered (since $\boldsymbol{B} \boldsymbol{i}_{\mathbf{1}}<\mathbf{0 . 1}$ the internal resistance to transferring heat is not effective or effects are considered negligible). Strong or large value of $\boldsymbol{B} \boldsymbol{i}_{\boldsymbol{1}}$ indicates effective internal diffusion resistance. From figure we can notice that both temperature profiles for magnetic/non-magnetic nanofluids increases for increasing values of $\boldsymbol{B} \boldsymbol{i}_{\mathbf{1}}$. Similar physical trend was observed in concentration boundary layer which indicates that momentum diffusion is larger than thermal diffusion and hence concentration profile increases with rising values of $\boldsymbol{B} \boldsymbol{i}_{2} . \mathbf{F i g}(\mathbf{6})$ depicts the influence of porosity parameter $(\mathrm{Kp})$ for velocity profile. It is obvious from figure the velocity distribution decelerates as we increase the porosity parameter which happens due to the presence of the porous media. Generally this porosity factor has the tendency to soak the significant amount of fluid from the boundary layer, so the velocity profile depreciates in the entire flow domain. Analytical results which incorporate with parameter of volume fraction $\phi$ were plotted in $\mathbf{F i g}(\mathbf{7})$ for two different sorts of nanoparticles named as $\mathrm{Fe}_{3} \mathrm{O}_{4}$ and $\mathrm{Al}_{2} \mathrm{O}_{3}$. It was perceived that velocity of nanofluids decelerate with increasing $\phi$ but increasing values of $\phi$ enhances the thermal profile. It is physically true due to the fact that when $\phi$ increases it enhances viscosity as well as thermal conductivity of the nanoparticles this accelerates the thermal profile and finally causes depreciation in velocity. According to Maxwell and Brickman [5] increasing in volume fraction increases the viscosity and thermal conductivity of nanofluids. This causes decrease in velocity. $\operatorname{Fig}(\mathbf{8})$ describe the effects of radiation parameter(R), heat sink / $\operatorname{source}\left(\boldsymbol{A}^{*}\right)$ and Viscous dissipation(Ec) factors for $\mathrm{Fe}_{3} \mathrm{O}_{4}$ and $\mathrm{Al}_{2} \mathrm{O}_{3}$. It was concluded that for increasing values of $\mathrm{R}, \mathrm{A}^{*}$ and Ec the magnetic and non-magnetic nanofluid profiles get enhanced. Scientifically, the heat flux existence causes the temperature higher so the thermal boundary layer for the whole flow region increases for increasing values of Rosseland diffusion application. By larger values of $\boldsymbol{A}^{*}$ which acts as a responsible factor of heat generation, releases more energy to the fluid and then this uplift the temperature profile. Also, the frictional heat will be generated as the fluid moves faster along the surface this causes hike in temperature boundary layer thickness. Fig(9) exhibits the behavior of Scmidth number, Soret number and chemical reaction parameter. The concentration profile depreciates for growing values of $\mathrm{Sc}$ and $\mathrm{K}_{r}$ for both $\mathrm{Fe}_{3} \mathrm{O}_{4}$ and $\mathrm{Al}_{2} \mathrm{O}_{3}$ but rising values of $\mathrm{Sr}$ 
parameter both the magnetic \& non-magnetic nanofluid profiles indicates the increasing trend. All these above behavior of concentration profiles are according to the existing physical situations. On explanatory note, the rate of mass transfer gets smaller when diffusion coefficient increases, this retards both the concentration profiles of magnetic and non-magnetic nanofluids. Similarly, higher molecular motion leads sharpen process of mass transportation which resulted the deceleration in the concentration profile. But increasing values of $\mathrm{Sr}$ both the $\mathrm{Fe}_{3} \mathrm{O}_{4}$ and $\mathrm{Al}_{2} \mathrm{O}_{3}$ profiles shows increasing trend. This indicates the physical reality due to the temperature gradient, mass fluxes move from the smaller to larger concentration species. This predicts that increasing the values of $\mathrm{Sr}$, diffusion species causes increment in concentration profiles.

\section{Conclusion}

The current investigation specifically discussed the mass and heat transfer analysis of Newtonian(Water) \& nonNewtonian(Sodium Alginate) suspension containing magnetic $\left(\boldsymbol{F} \boldsymbol{e}_{\mathbf{3}} \boldsymbol{O}_{\mathbf{4}}\right)$ \& non-magnetic $\left(\boldsymbol{A l}_{\mathbf{2}} \boldsymbol{O}_{\mathbf{3}}\right)$ nanoparticles . The unsteady convective flow of casson nanofluid along a non-linear stretched sheet by permeable media under the effects of magnetic field, chemical reaction, thermo-diffusion, viscous and ohmic dissipation was thoroughly discussed in this work. Some imperative results are listed as under:

- The velocity, temperature and concentration profiles decreases for increasing values of unsteady parameter $(\mathrm{S})$. Almost similar results were seen for both magnetic \& non-magnetic nanoparticles.

- The velocity profile depreciate by increasing magnetic field(M), slip factor $(\boldsymbol{\delta})$, porosity parameter $\left(\boldsymbol{K}_{\boldsymbol{p}}\right)$, volume fraction $(\boldsymbol{\phi})$ and casson $\operatorname{parameter}(\boldsymbol{\beta})$.

- Magnetic field(M), thermal radiation(R), heat sink / source parameter $\left(\boldsymbol{A}^{*}\right)$, Biot number $\left(\boldsymbol{B} \boldsymbol{i}_{\mathbf{1}}\right)$, volume fraction $(\phi)$ and viscous dissipation(Ec) enhance the thermal profile.

- The concentration profile starts decreasing for increasing Schmidth number(Sc) and chemical reaction parameter $\left(\boldsymbol{K}_{\boldsymbol{r}}\right)$ but increases for increasing values of slip parameter $\left(\boldsymbol{B i}_{\mathbf{2}}\right)$ and Soret number $(\mathrm{Sr})$.

- It was also observed that the performance of non-Newtonian(Sodium Alginate) fluid in heat and mass transfer is better than Newtonian(water) based fluid.

- No major difference was seen in heat and mass transfer when compared with Magnetic nanoparticles and Non-magnetic nanoparticles.

- It would be worth mentioning that all physical behavior of this flow problem coincides very well with already published literature either graphical or in tabular representation.

\section{Declaration}

- Data Availability Statement: All the data set for supporting the results and conclusion is provided in the manuscript.

- Competing interests: All the authors declared no Competing interests.

- Funding Statement: The research and publication of this article is self-funded and did not receive any specific funding.

- Authors Contribution: a). Ms. Kanwal Jabeen (Corresponding Author) designed the experiments and wrote the manuscript

b). Prof. Dr. Muhammad Mushtaq (Supervisor)was in charge of the whole work.

c). Mr. Rana Muhammad Akram Muntazir (Author) assisted with structure and language of the manuscript. All authors read and approved the fnal manuscript.

- Acknowledgement The authors sincerely thank to "University of Engineering and Technology Lahore Pakistan". 


\section{References}

[1] N. Casson, Rheology of Dispersed System, Vol. 84, Pergamon Press, Oxford, UK, 1959.

[2] S.U.S. Choi, Enhancing thermal conductivity of fluids with nanoparticles Proceedings, ASME International Mechanical Engineering Congress and Exposition, San Fransisco, USA, ASME Fluids Eng Div, 231/MD 66 (1995), pp. 99-105

[3] K. A. Yih, Free convection effect on MHD coupled heat and mass transfer of a moving permeable vertical surface. Int. Commun. Heat Mass Transf. 26(1), pp. 95-104, 1999.

[4] S. Choi, Z.G. Zhang, W. Yu, F.E. Lockwood, E.A. Grulke Anomalous thermal conductivity enhancement on nanotube suspensions, Appl. Phys. Lett., 79 (2001), pp. 2252-2254

[5] Eastman J. A., Choi S. U. S., Li S., Yu W. and Thompson L. J. . Anomalously increased effective thermal conductivities of ethylene glycolbased nanofluids containing copper nanoparticles. Applied physics letters. 78(6): 2001, Pp.718-720.

[6] J. Buongiorno, Convective transport in nanofluids, J. Heat. Transfer 128 (2006) 240250.

[7] J. Boungiorno, A benchmark study of thermal conductivity of nanofluids, J. Appl.Phys. 106 (2009) 094312.

[8] S.C. Moon, B.-Y. Ryu, J.K. Choi, B.W. Jo, R.J. Farris, The morphology and mechanical properties of sodium alginate based electrospun poly(ethylene oxide)nanofibers, Polym. Eng. Sci. 49 (1) (January 2009) 5259 .

[9] A. J. Chamkha, A. M. Aly, and M. A. Mansour, Similarity solution for unsteady heat and mass transfer from a stretching surface embedded in a porous medium with suction/injection and chemical reaction effects, Chemical Engineering Communications, 197( 6), 2010 pp. 846858

[10] R. Ellahi, A. Zeeshan, K. Vafai, H.U. Rahman, Series solutions for magnetohydrodynamic flow of nonNewtonian nanofluid and heat transfer in coaxial porous cylinder with slip conditions, Proc. IMechE. N. J. Nanoeng. Nanosys 225 (3) 2011) 123132.

[11] T.G. Motsumi, O.D. Makinde, Effects of thermal radiation and viscous dissipation on boundary layer flow of nanofluids over a permeable moving flat plate, Phys. Sci. 86 (2012) 045003.

[12] Aurangzaib, A. R. M. Kasim, N. F. Mohammad, and S. Shafie, "Effect of thermal stratification on MHD free convection with heat and mass transfer over an unsteady stretching surface with heat source, Hall current and chemical reaction," Int. J. Adv. Eng. Sci. Appl. Math. 4(3), 2012, pp. 217-225

[13] Colla L.and Fedele L., Scattolini M. and Bobbo S. Water-based Fe2O3 nanofluid characterization: thermal conductivity and viscosity measurements and correlation. Advances in Mechanical Engineering. 4(1), 2012

[14] S. Mukhopadhyay, "Casson fluid flow and heat transfer over a nonlinearly stretching surface, Chin. Phys. B, 22( 7), 2013

[15] S. Mukhopadhyay, "Analysis of boundary layer flow over a porous nonlinearly stretching sheet with partial slip at the boundary, Alexandria Eng.J. 52(4),2013 pp. 563-569,

[16] Ram P. and Kumar V. Swirling Flow of Field Dependent Viscous Ferrofluid Over a Porous Rotating Disk with Heat Transfer. International Journal of Applied Mechanics. 6(04), 2014, 1450033.

[17] S. Nadeem, R. Ul Haq, and N. S. Akbar, MHD three-dimensional boundary layer flow of Casson nanofluid past a linearly stretching sheet with convective boundary condition," IEEE Trans. Nanotechnol. 13(1), pp. $1326-1332,2014$.

[18] Qasim M, Khan ZH, Khan WA, Ali Shah I (2014) MHD Boundary Layer Slip Flow and Heat Transfer of Ferrofluid along a Stretching Cylinder with Prescribed Heat Flux. PLoS ONE 9(1): e83930. doi:10.1371/journal.pone.0083930

[19] Sheikholeslami M. and Ganji D. D. Ferrohydrodynamic and magnetohydrodynamic effects on ferrofluid flow and convective heat transfer. Energy. 75(1),2014, Pp: 400-410.

[20] Sheikholeslami M., Ganji D. D. and Rashidi M. M. Ferrofluid flow and heat transfer in a semi annulus enclosure in the presence of magnetic source considering thermal radiation. Journal of the Taiwan Institute of Chemical Engineers. 47(1),2015, Pp: 6-17. 
[21] Aaiza Gul1 et al. Heat Transfer in MHD Mixed Convection Flow of a Ferrofluid along a Vertical Channel. PLOS ONE. 2015(1), 2015 Pp.1-14

[22] I. S. Oyelakin, S. Mondal, and P. Sibanda, "Unsteady Casson nanofluid flow over a stretching sheet with thermal radiation, convective and slip boundary conditions, Alexandria Eng. J., 55(2) 2016, pp. 1025-1035

[23] S.Nadeem et al. Mathematical analysis of ferromagnetic fluid embedded in a porous medium. Results in Physics7(1), 2017, Pp. 2361-2368

[24] A.K. Abdul Hakeem, S. Saranya, B. Ganga, Comparative study on Newtonian/non-Newtonian base fluids with magnetic/non-magnetic nanoparticles over a flat plate with uniform heat flux, J. Mol. Liq. 230 (2017) 445452 .

[25] S. Saranya, P. Ragupathi, B. Ganga, R.P. Sharma, A.K. Abdul Hakeem, Non-linear radiation effects on magnetic/non-magnetic nanoparticles with different base fluids over a flat plate. Advanced Powder Technology 29 (2018) Pp.19771990

[26] A. Khanet al. MHD Flow of Sodium Alginate-Based Casson Type Nanofluid Passing Through A Porous Medium With Newtonian Heating. Sci Rep 8, 8645 (2018). 1-11

[27] F. Mabood and S. Shateyi. Multiple Slip Effects on MHD Unsteady Flow Heat and Mass Transfer Impinging on Permeable Stretching Sheet with Radiation. Modelling and Simulation in Engineering 2019,1- 11

[28] I. Ullah et al. Unsteady Free Convection Flow of Casson Nanofluid Over a Nonlinear Stretching Sheet. IEEE Access 7(1) 2019

[29] K.Jabeen, Mushtaq M and Akram R.M. A comparative study of MHD flow Analysis in a Porous Medium by Using Differential Transformation Method and Variational Iteration Method. Journal of Contemporary Applied Mathematics. 9(2) (2019)

[30] J.A.Gbadeyan., E.O.Titiloye, A.T.Adeosun. Effect of variable thermal conductivity and viscosity on Casson nanofluid flow with convective heating and velocity slip. Heliyon 6(1) 2020 Pp. 1-10

[31] Firas A.Alwawi et al. MHD natural convection of Sodium Alginate Casson nanofluid over a solid sphere. Results in Physics16(1), 2020, 1-12

[32] K.Jabeen, Mushtaq M and Akram R.M. Analysis of MHD boundary layer flow over a non-linear stretching sheet in a porous medium using semi analytical approaches. Journal of Mathematical problems in Engineering 2020(1) (2020) 1-9

[33] K. Jabeen, M. Mushtaq, and R. M. Akram Muntazir. Analysis of MHD Fluids around a Linearly Stretching Sheet in Porous Media with Thermophoresis, Radiation, and Chemical Reaction. Mathematical Problems in Engineering. 2020(1),2020 Pp.1-14 
Figures

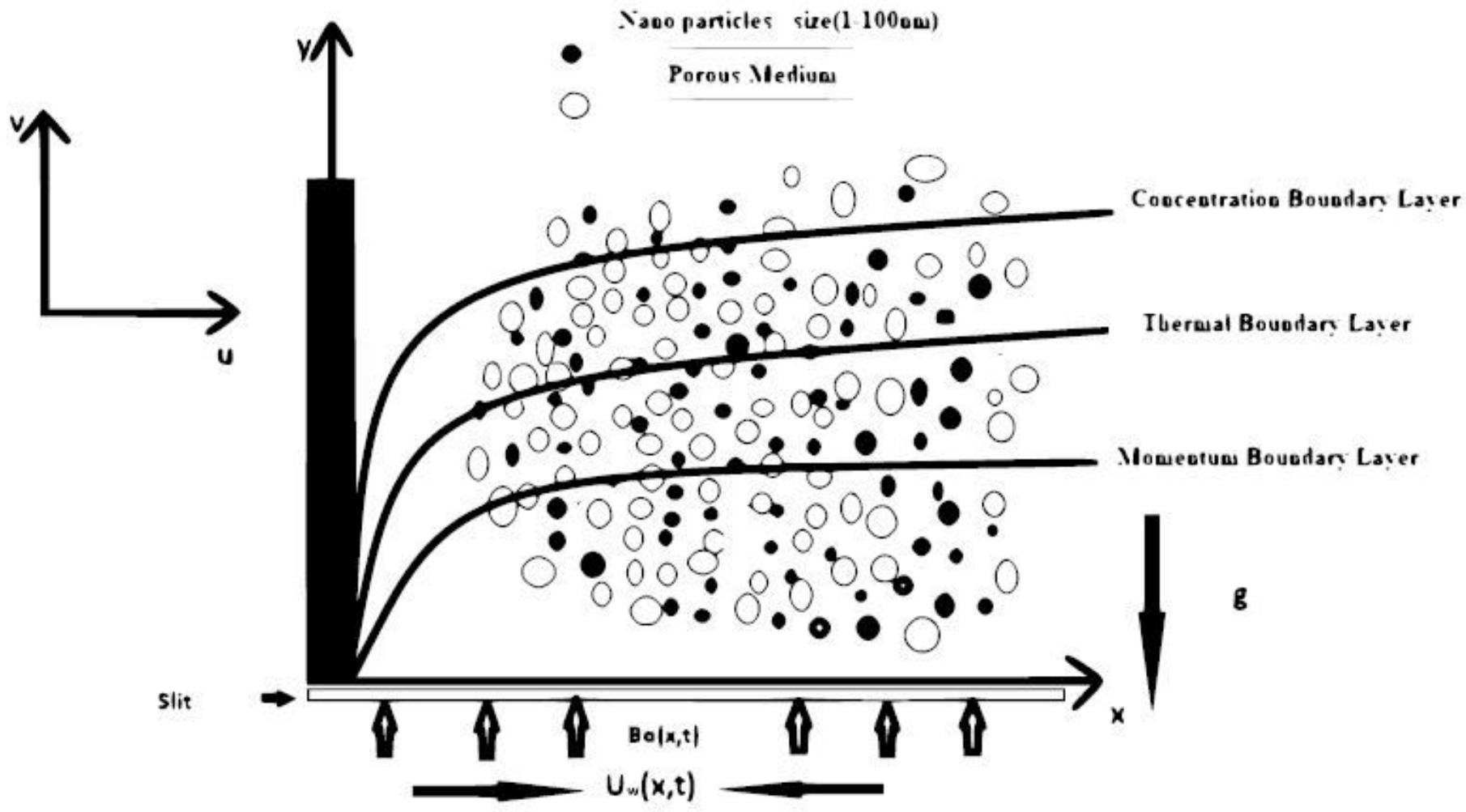

Figure 1

Physical Interpretation of Flow Model 

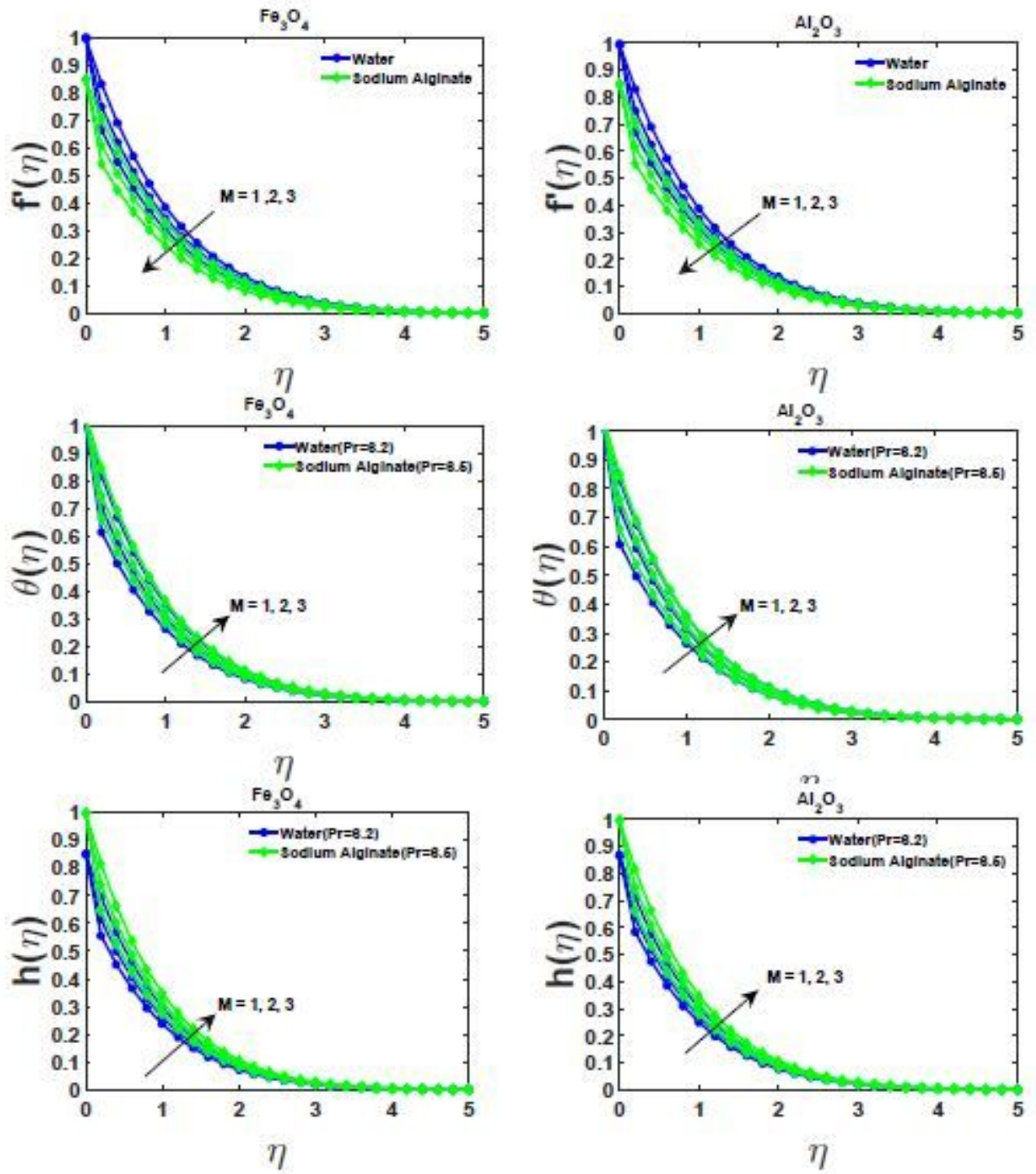

Figure 2

Effects of Magnetic field M for Fe3O4 and Al2O3 

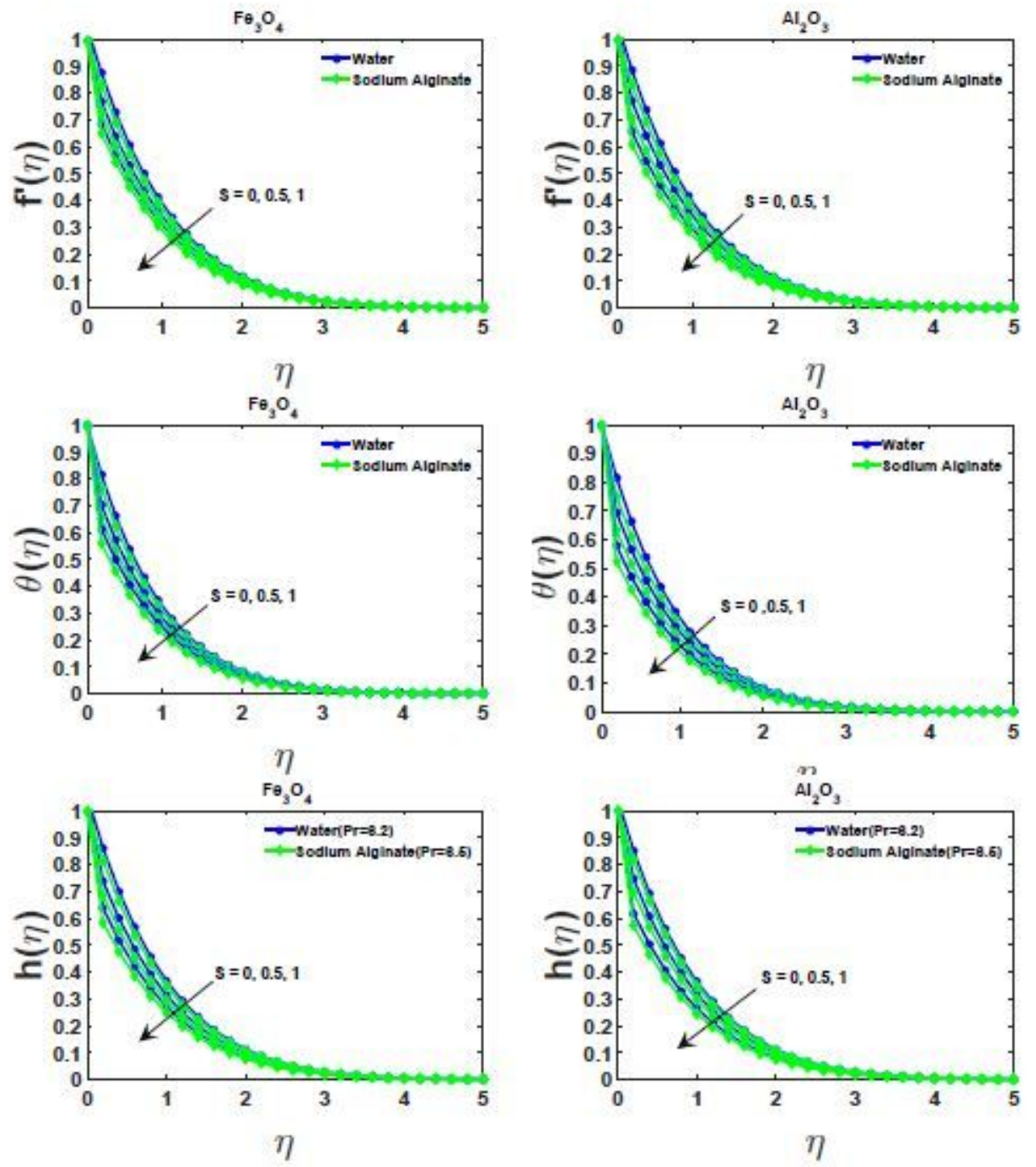

Figure 3

Effects of Unsteady parameter S for Fe3O4 and Al2O3
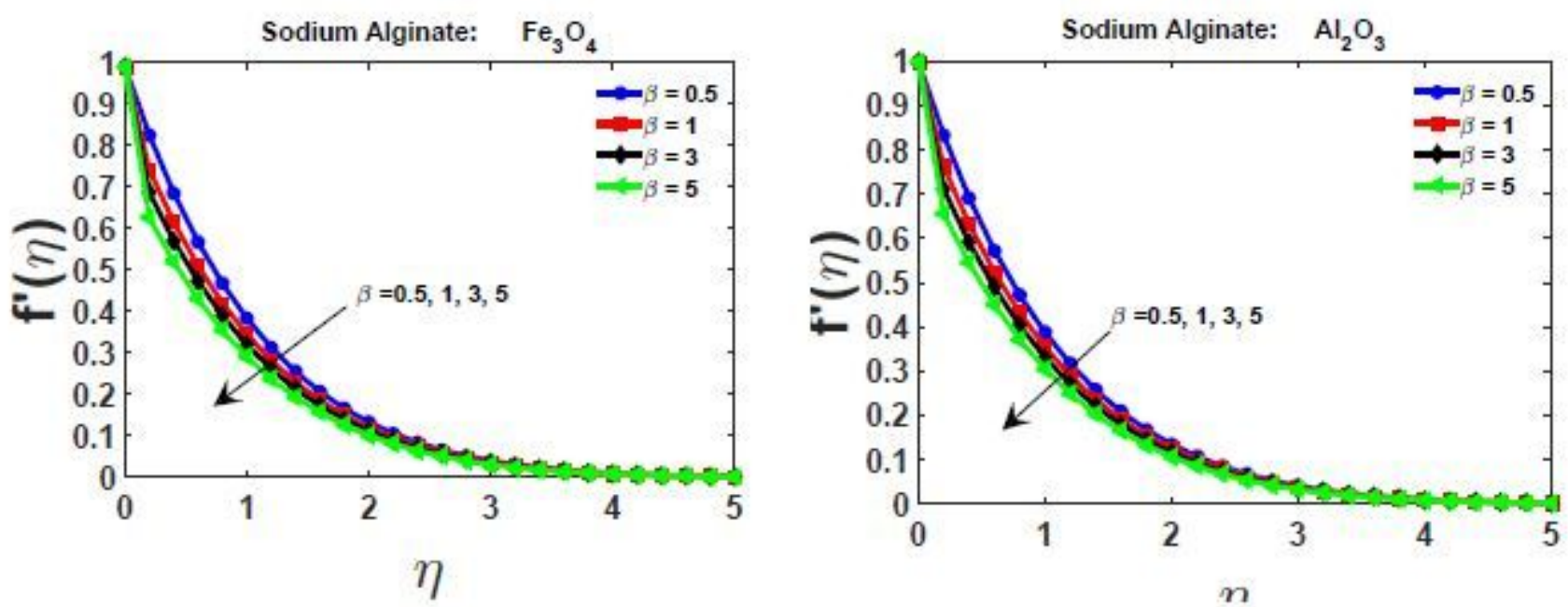
Figure 4

Effects of Casson Parameter $\beta$ for $\mathrm{Fe} 3 \mathrm{O} 4$ and $\mathrm{Al} 2 \mathrm{O} 3$
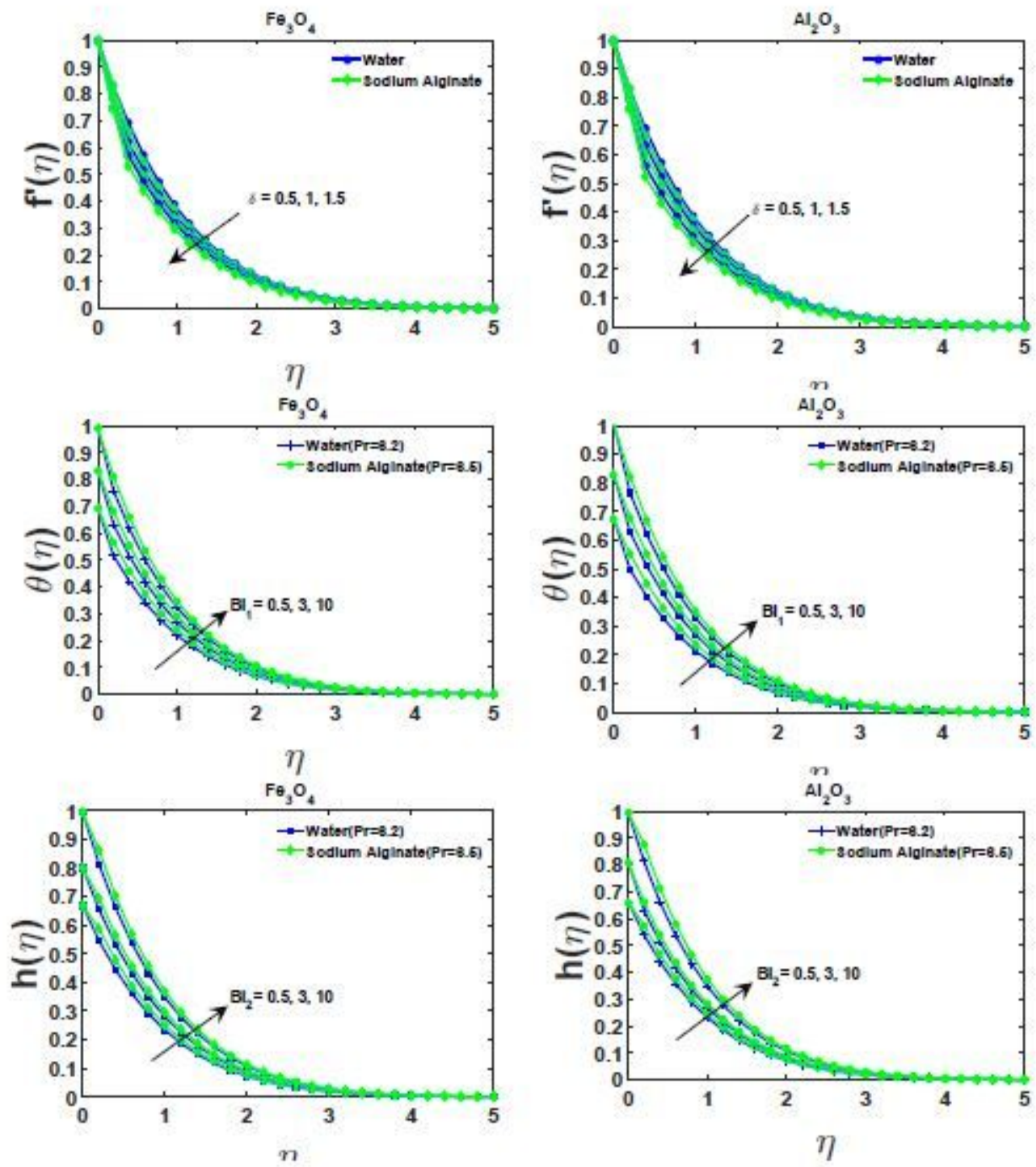

Figure 5

Effects of Slip Parameters $\delta, \mathrm{Bi} 1$ and Bi2 for Fe3O4 and Al2O3 

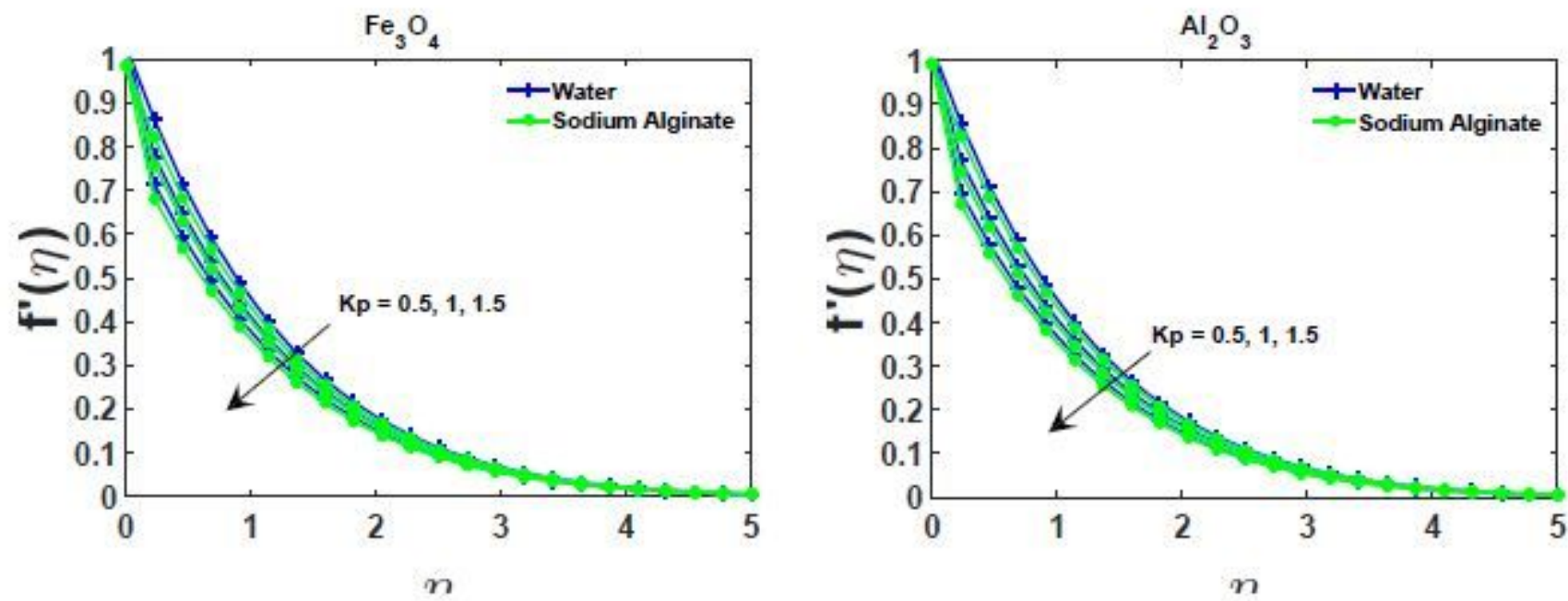

Figure 6

Effects of Porosity Parameters Kp for Fe3O4 and Al2O3
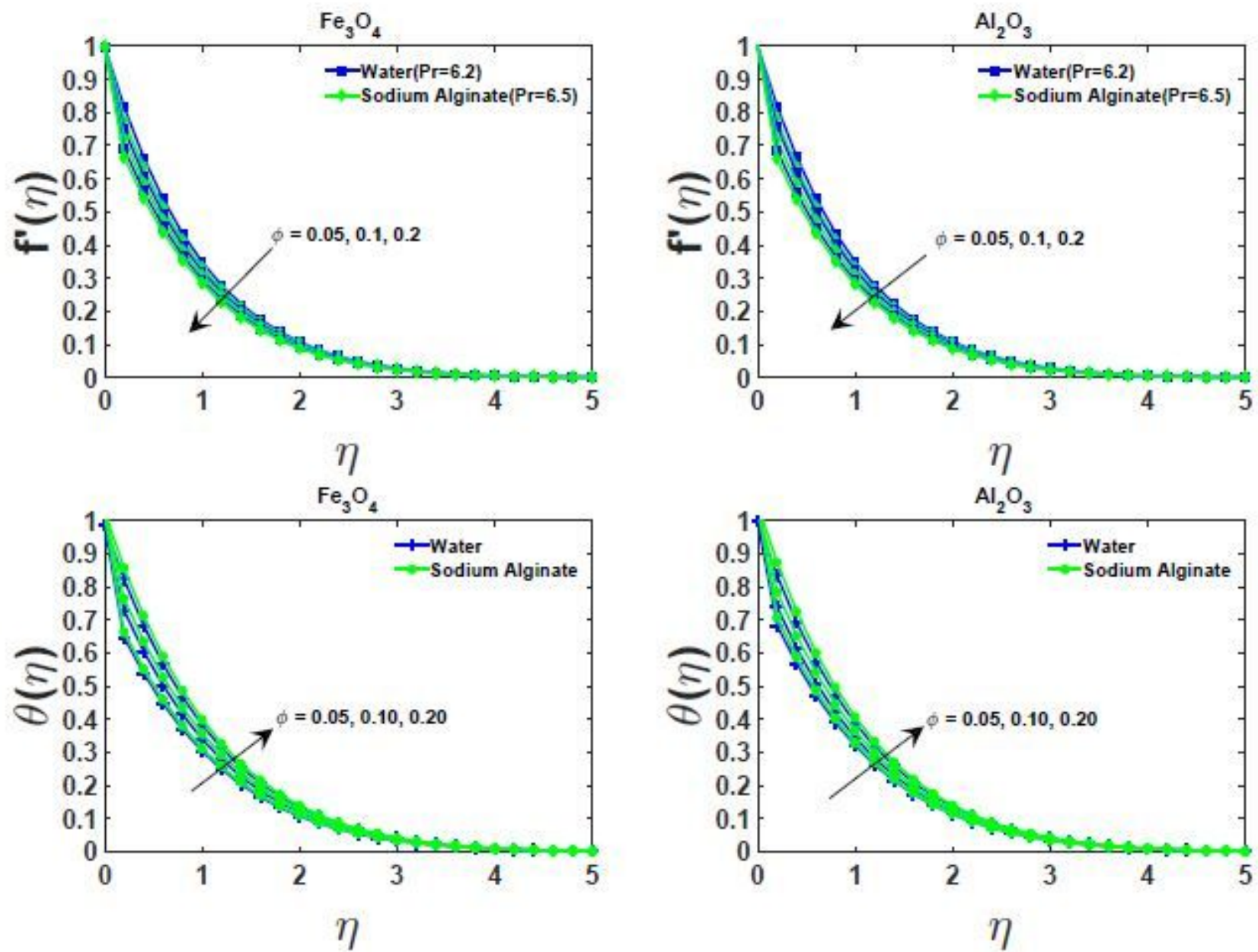

Figure 7 

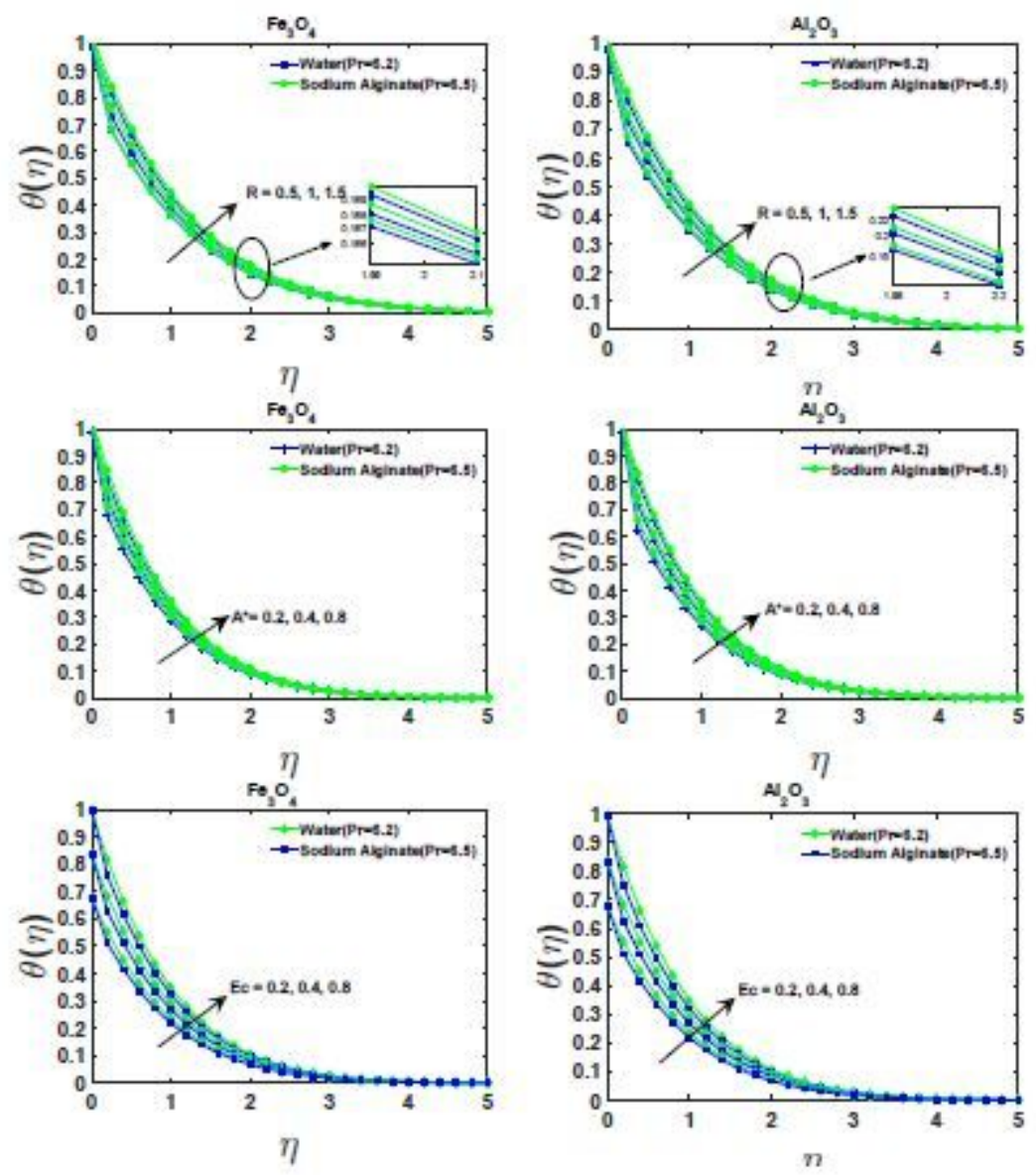

Figure 8

Effects of Radiation Parameters(R), heat sink / source(A囚) and Viscous dissipation(Ec) factors for Fe304 and $\mathrm{Al} 2 \mathrm{O} 3$ 

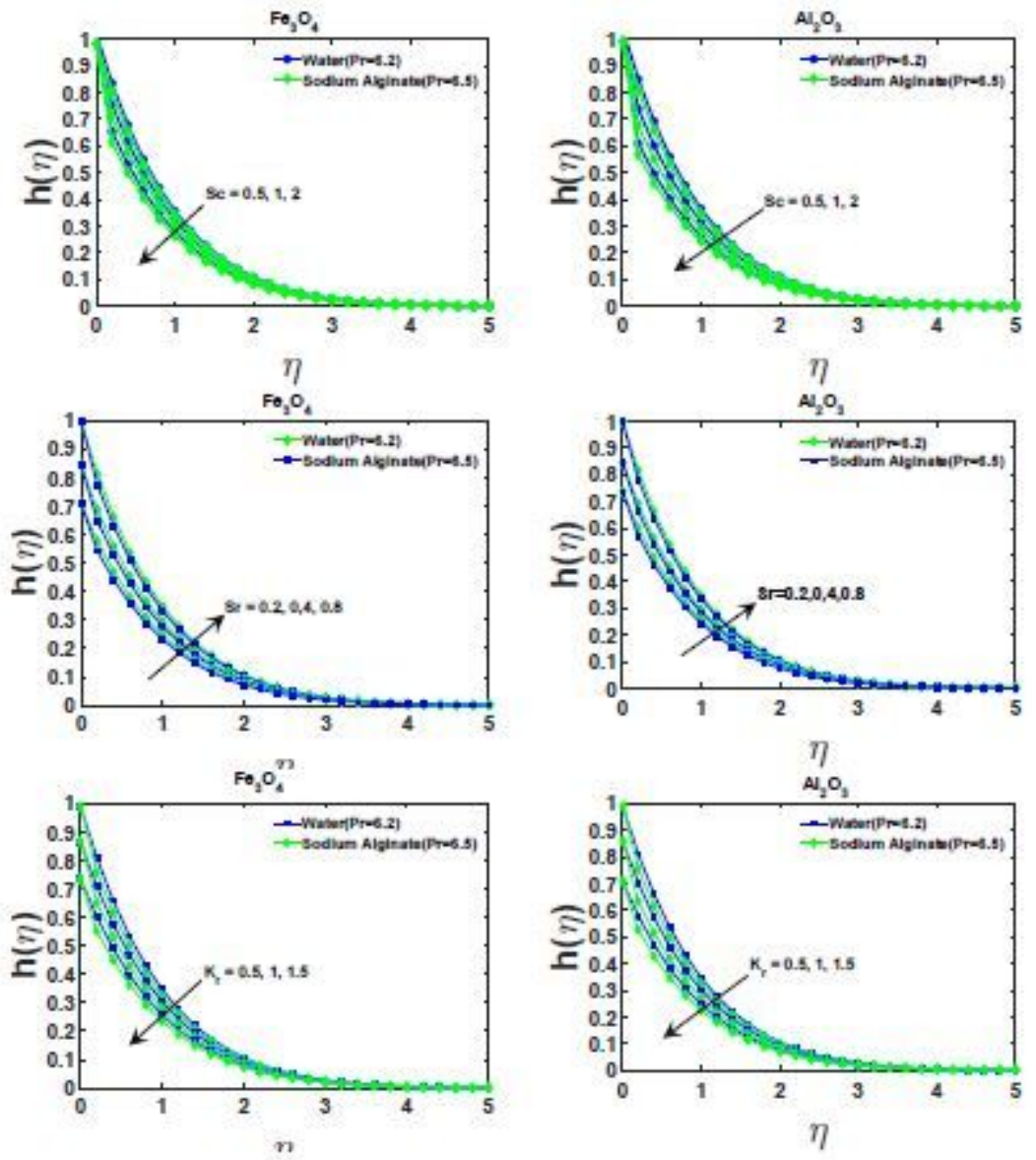

Figure 9

Effects of Sc, Sr and Kr for Fe3O4 and Al2O3 